\title{
Auxiliary Antenna-Assisted Interference Mitigation for Radio Astronomy Arrays
}

\author{
Brian D. Jeffs, Senior Member, IEEE, Lisha Li, and Karl F. Warnick, Member, IEEE
}

\begin{abstract}
Interferometric image synthesis in radio astronomy is plagued by signal corruption from man-made sources. The very weak signals of interest can be overwhelmed by such interference. Recent work has proposed using array signal processing techniques of adaptive beamforming, adaptive filtering, and subspace projection to remove interference prior to image synthesis. In some practical scenarios, we have found poor cancellation performance when using such methods. Because signal-of-interest levels in radio astronomy (RA) are usually well below the noise, even interference to noise ratios (INR) less than unity can affect signal estimation. At these low INRs, it is difficult to estimate interference parameters or statistics with sufficient accuracy for high-performance adaptive cancellation or subspace projection. By adding a few (one to three) low-gain (relative to the primary telescope dishes) "auxiliary" antennas to an array, it is possible to overcome this problem. This paper will show that using such antennas (e.g. commercial-grade 3-m dishes) with an existing array can significantly improve interference rejection. New extensions to subspace projection spatial filtering methods are presented, along with analytical and simulated results for performance comparison.
\end{abstract}

Index Terms-Adaptive arrays, interference cancellation, radio astronomy, spatial filtering, subspace projection.

\section{INTRODUCTION}

$\mathbf{T}$ HIS paper considers signal subspace projection methods for interference removal in large radio astronomy (RA) imaging arrays, such as the Very Large Array (VLA) at Socorro, NM. Specifically, we propose that including a few relatively low-gain, interferer tracking, auxiliary antennas in the array can significantly improve interference rejection. Relative performance under these conditions for four algorithms (including new extensions to existing subspace projection methods) is evaluated. Although a variety of man-made sources plague RA, we are particularly interested in canceling interfering downlink signals from satellites such GLONASS and IRIDIUM. These very strong sources affect critical RA observation spectral bands. For example, the Russian GLONASS signal produces strong spectral sidelobes that extend through the important hydroxyl ion $(\mathrm{OH})$ emission band [1]-[4]. Satellite sources can be problematic because they are not in fixed locations and often rapidly traverse both the mainlobe and sidelobes of the telescope beam. This nonstationary interference requires an adaptive processing approach for effective mitigation.

Manuscript received February 4, 2003; revised February 22, 2004. This work was supported by National Science Foundation Grant AST-9987339. The associate editor coordinating the review of this paper and approving it for publication was Dr. Constantinos B. Papadias.

The authors are with the Department of Electrical and Computer Engineering, Brigham Young University, Provo, UT 84602 USA (bjeffs@ee.byu.edu; lisha@ee.byu.edu; warnick@ee.byu.edu).

Digital Object Identifier 10.1109/TSP.2004.840787
There are many existing and proposed RA interference mitigation techniques, including time gating to blank impulsive interference [5], [6], adaptive frequency-selective filtering [7], parametric signal subtraction [2], deterministic null forming [8], adaptive beamforming [9], temporal adaptive filtering [1], [7], [10], frequency domain post-correlation processing with a reference antenna [11], and spatial filtering by subspace projection [12]-[14]. In addition, Thompson showed that during the long integration times needed for RA, interference that moves relative to the instantaneous array pointing direction is attenuated due to a decorrelation effect caused by averaging over many interferometric "fringe rotations" [15].

The subspace projection approach was adapted to RA imaging by Leshem et al. [12]-[14]. This is one of the most promising methods for removing very strong satellite downlink interference and is the starting point for the algorithms and analysis presented in this paper. Our preliminary work is reported in [16]. From a historical perspective, the fundamental idea of excising interference from a desired signal by vector subspace partitioning has appeared in many forms for a variety of disciplines and applications, including adaptive beamforming, subspace detectors, and interference cancellers used in SONAR and RADAR. For examples, see [17]-[23].

In many practical RA imaging scenarios, we have found poor cancellation performance with adaptive array processing and subspace projection methods. Shallow, unstable beamforming nulls and poor interferer subspace estimates are common. This is usually due to the relatively low interference-to-signal-plusnoise ratio (ISNR) at antenna feeds of the high gain antennas used in imaging arrays. This problem occurs even when interfering sources have flux densities that are many tens of decibels higher than the desired signal because of the low sidelobe response for the high gain antennas. It must be remembered that in RA, signals of interest are usually well below the noise floor, so an interference level at antenna feeds that is much higher than the signal may not be significantly higher than the noise. In this case, it is difficult to form the accurate subspace estimates necessary for high-performance mitigation. In other words, the interference is often sufficiently strong to corrupt signal reception but not sufficiently higher than noise to permit effective interference rejection.

By adding a few auxiliary antennas with modest gain to an existing imaging array, and by developing or modifying subspace projection algorithms to exploit this additional information, interference mitigation can be significantly improved. These auxiliaries can, for example, be small dishes steered to track a satellite. Very low noise temperature receivers are not required. The fundamental idea here is to obtain a high ISNR copy of the un- 


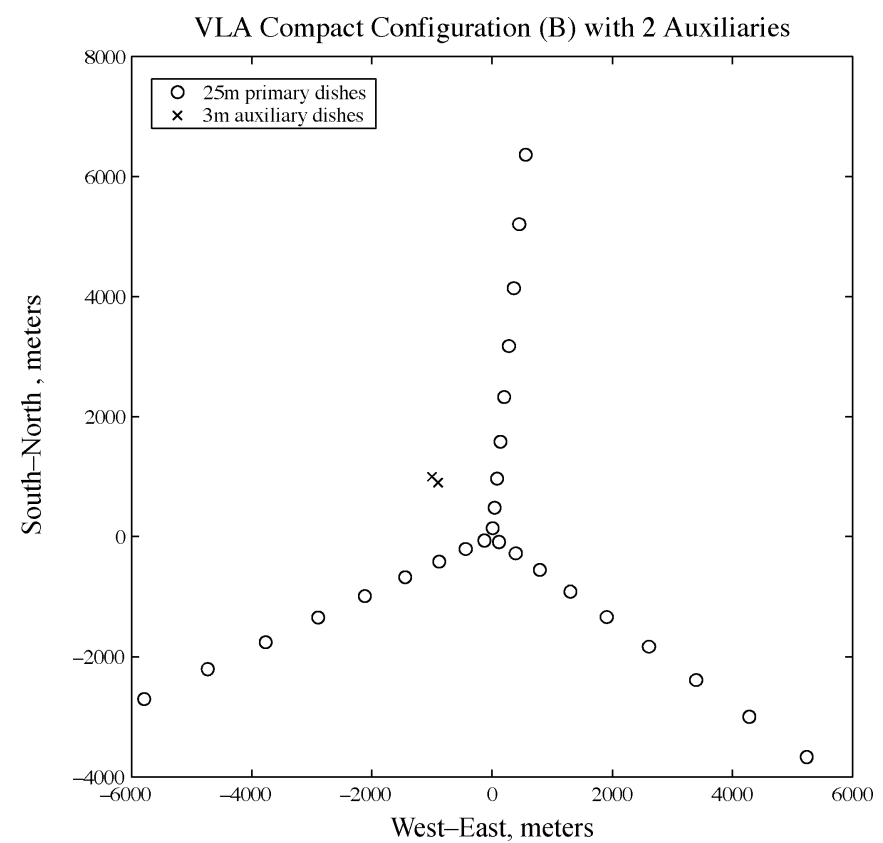

Fig. 1. Array element geometry for the VLA configuration B, showing possible location for two auxiliary 3-m diameter dishes.

desirable signal and use this to improve covariance matrix (and thus subspace) estimation in the cancellation or excision algorithm of choice. This paper will show that such an approach can improve interference rejection by tens of decibels. Some previous work has involved use of auxiliary or reference antennas with single telescopes [1], [7], [10], [11], whereas we will focus on improvements to subspace projection methods suited to large arrays.

As an example, Fig. 1 illustrates how the VLA can be augmented with two auxiliary antennas for use with the algorithms described below. The array geometry shown is for the so-called "B" configuration, and the " $\times \mathrm{s}$ " indicate two proposed (arbitrary) auxiliary locations. This array geometry will be used in all simulations below. The current VLA facility includes, at (very roughly) the indicated location, a 3-m satellite tracking dish and an omnidirectional antenna used for interference studies, although they are not currently connected to the correlator.

\section{RAdio Imaging ARray Signal Model}

We present here a very brief simplified discussion of techniques used in conventional RA synthesis imaging. The signal structure will be described, and needed notation will be introduced. More detailed treatments are found in [14], [26], and [27]. Fig. 2 illustrates many of the terms defined below.

Consider an $M$ element imaging antenna array with the $n$th complex baseband data sample (across the array) represented by

$$
\begin{aligned}
\mathbf{x}[n] & =\left[x_{1}[n], \cdots, x_{M}[n]\right]^{T} \\
& =\mathbf{s}[n]+\mathbf{i}[n]+\eta[n]
\end{aligned}
$$

where $\mathbf{s}, \mathbf{i}$, and $\eta$ correspond to the narrowband signal from a desired deep space object, man-made (e.g. satellite) interference, and noise, respectively. The array covariance matrix is given by

$$
\mathbf{R}=E\left\{\mathbf{x}[n] \mathbf{x}^{H}[n]\right\},=\mathbf{R}^{s}+\mathbf{R}^{i}+\mathbf{R}^{\eta}
$$
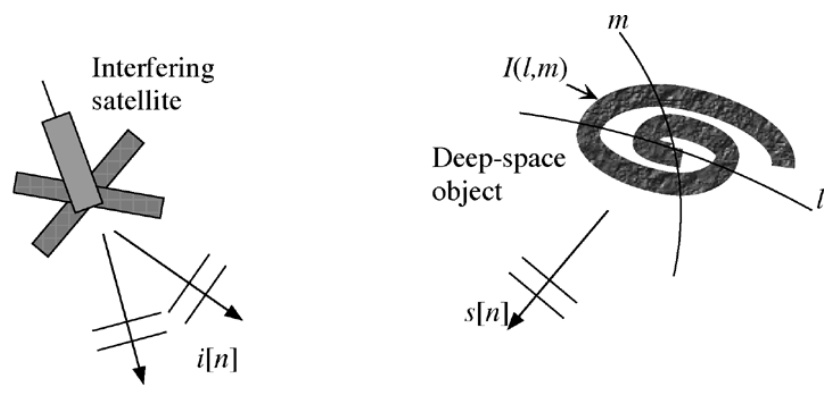

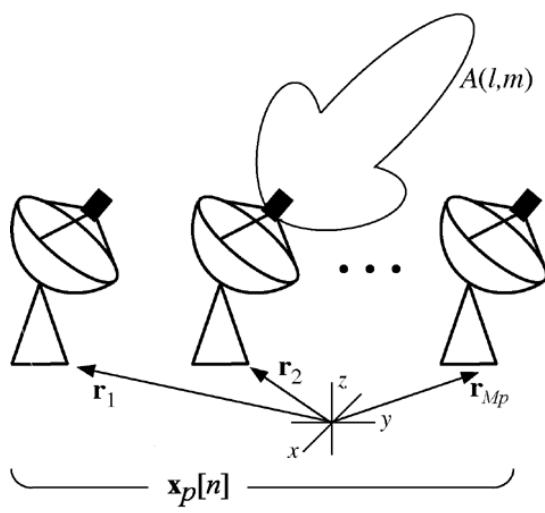

High gain primary array

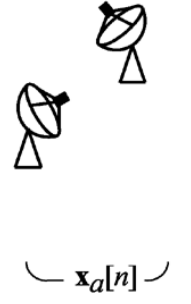

Low gain auxiliaries
Fig. 2. Schematic view of signals, sensors, and notation used for RA synthesis imaging in the presence of satellite interference.

where $E\{\}$ is expectation, and ${ }^{H}$ indicates matrix conjugate transpose. A sample estimate $\hat{\mathbf{R}}$ is formed by a real-time correlator at the array. In radio imaging, this spatial cross-correlation between array element pairs comprises the essential image forming information. Elements of the signal-of-interest covariance matrix $\mathbf{R}^{s}$ (also called "visibilities") correspond to spatial frequency domain image samples of the desired object, with discrete frequency values determined by the physical vector distances (called baselines) between all combinations of antenna pairs. In the absence of noise or interference, this Fourier relationship is expressed by the visibility function [26]

$$
V(u, v)=\iint I(l, m) A(l, m) e^{-j 2 \pi(u l+v m)} d l d m
$$

where $I(l, m)$ is the desired source intensity image as a function of sky position coordinates $l, m, A(l, m)$ is the antenna beam response pattern for a single array element $(A(l, m) \approx 1$ for compact objects contained in the beam mainlobe), and $u$ and $v$ represent the spatial frequencies in the directions of perpendicular image coordinate axes $l$ and $m$, respectively. The relationship between elements of $\mathbf{R}^{s}$, samples of $V(u, v)$, and the corresponding baseline vectors from array element pairings is given by

$$
\begin{aligned}
\left\{\mathbf{R}^{s}\right\}_{(i, k)} & =V\left(u_{i k}, v_{i k}\right), \text { where } \\
{\left[u_{i k}, v_{i k}, w_{i k}\right]^{T} } & =\mathbf{T}\left(\mathbf{r}_{k}-\mathbf{r}_{i}\right) .
\end{aligned}
$$

$\mathbf{r}_{i}$ and $\mathbf{r}_{k}$ are position vectors for the $i$ th and $k$ th array elements, respectively, in the Earth coordinate system $(x, y, z)$, using units of wavelengths at the narrowband source frequency $f_{c}$. $\mathbf{T}$ is a coordinate transformation matrix that rotates $(x, y, z)$ into the $(u, v, \varphi)$ system, where $u$ is parallel to $l, v$ is parallel to $m$, and 
$\varphi$ is in the direction of the center of the space object of interest. $\left\{\mathbf{R}^{s}\right\}_{(i, k)}$ indicates the element in the $i$ th row and $k$ th column of $\mathbf{R}^{s}$.

A simplified image synthesis approach consists of, first, estimating $\mathbf{R}^{s}$ for a sufficiently large number of unique baseline position pairs, second, interpolating from these arbitrarily spaced observation to obtain a set of dense rectangular grid samples for $V(u, v)$, and, third, using a 2-D inverse FFT to solve (3) for $\hat{I}(l, m)$ (where indicates an estimated quantity). $\hat{\mathbf{R}}^{s}$ is usually recomputed periodically to exploit the Earth's rotation and thus yield correlation estimates for interelement baselines vectors with differing orientations in the $(u, v, w)$ system as $\mathbf{T}$ changes with time. This fills in the image frequency domain sample space $\left(u_{i k}, v_{i k}\right)$ with more baselines than can be obtained from a single $\hat{\mathbf{R}}^{s}$.

Cleary, an unbiased estimate of $\mathbf{R}^{s}$ is needed to avoid corrupting the image. As seen in (2), $\mathbf{R}^{s}$ may be observed only through the sample estimate $\hat{\mathbf{R}}$, which is corrupted by $\mathbf{R}^{i}, \mathbf{R}^{\eta}$, and sample estimation error. This paper presents adaptive interference removal methods for reducing the bias introduced by $\mathbf{R}^{i}$.

An augmented imaging array consists of $M_{p}$ high gain "primary" telescope antennas and $M_{a}$ relatively lower gain "auxiliary" antennas. The full array sample vector is

$$
\mathbf{x}[n]=\left[\begin{array}{l}
\mathbf{x}_{p}[n] \\
\mathbf{x}_{a}[n]
\end{array}\right]
$$

where $\mathbf{x}_{p}[n]$ and $\mathbf{x}_{a}[n]$ are signal vectors from primary and auxiliary arrays, respectively, as seen in Fig. 2. It is assumed that the auxiliary antennas have a much higher interference-to-signalplus-noise ratio (ISNR) than the primaries. These requirements can be met with very low cost antennas, positioners, and receivers (for example, we have used 3-m commercial L band satellite tracking dish antennas at a total system cost less than $\$ 7000$ per antenna). The augmented array covariance matrix has the following block structure:

$$
\mathbf{R}=\left[\begin{array}{ll}
\mathbf{R}_{p p} & \mathbf{R}_{p a} \\
\mathbf{R}_{a p} & \mathbf{R}_{a a}
\end{array}\right]
$$

where, for example, $\mathbf{R}_{a p}=E\left\{\mathbf{x}_{a}[n] \mathbf{x}_{p}^{H}[n]\right\}$.

\section{Algorithms FOR INTERFERENCE REMOVAL FROM CORRELATION DATA}

We will compare performance of four algorithms, including a new approach and extensions to previously proposed methods, as listed below.

\section{Subspace projection without auxiliaries $(S P)$}

This method was introduced to RA imaging by Leshem and van der Veen [12] and further developed in [13] and [28]. Only the original primary array antennas are used. This algorithm is included for comparison with auxiliaryassisted approaches.

Subspace projection with auxiliary antennas (SPA)

This is based on SP, except the array is augmented with auxiliary antennas. SP is performed on the entire augmented array.

Array multiple sidelobe canceller (MSC)
This method uses one or more auxiliaries and is derived from the classical multiple sidelobe canceller adaptive beamformer [29].

Auxiliary-assisted cross subspace projection (CSP)

This proposed approach uses only cross correlations between auxiliary antennas and the primary array to form better interference subspace estimates.

\section{A. Subspace Projection for Interference Mitigation}

All algorithms discussed in this paper use as input data the sample estimates of $\mathbf{R}$ [or its submatrices as structured in (6)], which are computed by a correlator processor, and they produce a reduced interference estimate of the desired signal covariance across the primaries $\hat{\mathbf{R}}_{p p}^{s}$. Due to rapid satellite interferer motion, sample covariances $\hat{\mathbf{R}}_{k}$ must be recomputed frequently using short-term integration periods over which the interferer array signature is relatively stationary:

$$
\begin{aligned}
\hat{\mathbf{R}}_{k} & =\frac{1}{N} \sum_{n=k N}^{(k+1) N-1} \mathbf{x}[n] \mathbf{x}^{H}[n], \quad 0 \leq k \leq K-1 \\
& =\mathbf{R}^{s}+\mathbf{R}_{k}^{i}+\mathbf{R}^{\eta}+\mathbf{E}_{k} .
\end{aligned}
$$

$N$ is the number of time samples in the short-term integration interval, $K$ is the number of short-term intervals that will be combined to form a final long-term projection bias corrected signal estimate, and $\mathbf{E}_{k}$ is sample estimation error due to finite $N . \mathbf{R}^{s}$ and $\mathbf{R}^{\eta}$ are assumed stationary over $N$ samples. A typical long-term integration time of $K N$ samples would correspond to (for example) $10 \mathrm{~s}$. Short-term integration times, corresponding to $N$ samples, are on the order of $10 \mathrm{~ms}$, which we have found to be appropriate for GLONASS signals seen at the VLA.

In each algorithm, a spatial filtering, or projection matrix $\mathbf{P}_{k}$ is derived from $\hat{\mathbf{R}}_{k}$ and used to block the interference component $\mathbf{R}_{k}^{i}$. It is assumed that $\mathbf{x}[n]$ is sufficiently bandlimited (by receiver bandpass filtering if sources are not intrinsically narrow) so that the narrowband array assumption is valid with respect to $\mathbf{i}[n]$. We require that $\mathrm{BW} \ll c / D$, where $\mathrm{BW}$ is the processing bandwidth, $c$ is the speed of light, and $D$ is the longest baseline length in the array. This is a tighter constraint than typically found in RA imaging where the signals of interest span a small angular sky patch and bulk time delay differences between antennas are corrected before correlation [26]. For the VLA configuration of Fig. 1, an appropriate worst-case is $\mathrm{BW}=5 \mathrm{kHz}$.

The filtered signal covariance estimate for a given short-term interval is

$$
\begin{aligned}
\hat{\mathbf{R}}_{k, p p}^{s} & =\mathbf{P}_{k} \hat{\mathbf{R}}_{k} \mathbf{P}_{k}^{H} \\
& \approx \mathbf{P}_{k}\left(\mathbf{R}^{s}+\mathbf{R}^{i}+\mathbf{R}^{\eta}\right) \mathbf{P}_{k}^{H} \\
& \approx \mathbf{P}_{k} \mathbf{R}^{s} \mathbf{P}_{k}^{H}+\mathbf{R}_{k}^{\eta \prime}
\end{aligned}
$$

where the approximation is due to neglecting sample error $\mathbf{E}_{k}$, $\mathbf{R}_{k}^{\eta \prime}=\mathbf{P}_{k} \mathbf{R}^{\eta} \mathbf{P}_{k}^{H}$, and the third line follows because $\mathbf{P}$ is designed to be (approximately) orthogonal to $\mathbf{R}_{k}^{i}$. When interference and signal subspaces are well separated, $\hat{\mathbf{R}}_{k, p p}^{s} \approx \mathbf{R}_{p p}^{s}$, but the projection can still introduce a bias. We will assume 
that $\mathbf{R}^{\eta}$ is diagonal or can be made so by prewhitening, as discussed in Section III-C. ${ }^{1}$ Without spatial filtering, a diagonal $\mathbf{R}^{\eta}$ would not seriously affect synthesis imaging, which relies on the off-diagonal terms of $\hat{\mathbf{R}}_{k, p p}^{s}$. However, $\mathbf{R}_{k}^{\eta^{\prime}}$ will have significant off-diagonal components. Both the off-diagonal noise and the projection bias in $\hat{\mathbf{R}}_{k, p p}^{s}$ can be cancelled with the inversion method described next.

Each $\mathbf{P}_{k}$ will introduce some bias in $\hat{\mathbf{R}}^{s}$ because the signal subspace is not entirely orthogonal to the interference subspace. Leshem et al. have proposed a method to cancel this bias [12], [13]. They showed that if motion is sufficient and $K>Q$, where $Q$ is the number of distinct interferes present, it is possible to invert a rank-enhanced, averaged version of $\mathbf{P}_{k}$ to form an unbiased signal covariance estimate

$$
\begin{aligned}
\hat{\mathbf{R}}^{s} & =\operatorname{unvec}\left\{\mathbf{C}^{\dagger} \operatorname{vec}\left(\sum_{k=0}^{K-1} \hat{\mathbf{R}}_{k, p p}^{s}\right)\right\} \\
\mathbf{C} & =\sum_{k=0}^{K-1}\left[\mathbf{P}_{k}^{*} \otimes \mathbf{P}_{\mathbf{k}}\right]
\end{aligned}
$$

where $\otimes$ indicates Kronecker matrix product, and * is complex conjugate. ${ }^{\dagger}$ denotes a thresholded matrix pseudo inverse that we have used here to improve stability and reduce noise amplification when $\mathbf{C}$ is ill conditioned by limited motion or integration times. $\mathbf{C}^{\dagger}$ is computed using singular value decomposition (SVD) with singular values smaller than $\operatorname{Tr}\left\{\hat{\mathbf{R}}^{s}\right\} / M_{p}$ forced to zero. $\operatorname{Tr}\{\}$ indicates matrix trace. This correction is used (when sufficient interferer motion is present) in all algorithms except MSC, which does not suffer from the projection bias problem.

\section{B. Subspace Projection Without Auxiliaries (SP)}

The spatial filtering method of Leshem et al. [12] and [13] is summarized here as background for the comparative analysis to follow. As originally described, only the primary data $\mathbf{x}_{p}[n]$ from high gain antennas is used. The interference component of $\mathbf{R}_{k, p p}$ spans a $Q$-dimensional subspace

$$
\begin{aligned}
\mathbf{R}_{k, p p}^{i} & =\mathbf{A}_{k, p} \mathbf{D} \mathbf{A}_{k, p}^{H} \\
\mathbf{A}_{k, p} & =\left[\mathbf{a}_{p, 1}, \cdots, \mathbf{a}_{p, Q}\right] \\
\mathbf{D} & =\operatorname{diag}\left\{\left[\sigma_{i, 1}^{2}, \cdots, \sigma_{i, Q}^{2}\right]\right\}
\end{aligned}
$$

where $\mathbf{a}_{p, q}$ is the primary array response vector for the $q^{\text {th }}$ interferer during the $k^{\text {th }}$ short-term integration interval, with power $\sigma_{i, q}^{2}$. The desired spatial filter is the perpendicular (relative to this interference subspace) projection matrix $\mathbf{P}_{k \text {,SP }}$, i.e., the rank $M_{p}-Q$ projector that satisfies $\mathbf{P}_{k, \mathrm{SP}} \mathbf{R}_{k, p p}^{i}=\mathbf{0}$. Subscript "SP" is used to distinguish $\mathbf{P}_{k, \mathrm{SP}}$ from corresponding terms in algorithms to follow.

If the array is accurately calibrated and interference arrival directions are known, then $\mathbf{A}_{k, p}$ is known, and the projection filter can be computed simply as

$$
\mathbf{P}_{k, \mathrm{SP}}=\mathbf{I}-\mathbf{A}_{k, p}\left(\mathbf{A}_{k, p}^{H} \mathbf{A}_{k, p}\right)^{-1} \mathbf{A}_{k, p}^{H} .
$$

${ }^{1}$ In RA, where extremely low noise cryogenic amplifiers are used, correlated noise, e.g. from the galactic center, can be seen above the independent thermal receiver noise.
In most cases, due to the uncalibrated gain and phase response in the antenna sidelobes where the interferer is seen, this level of detailed knowledge is not available, and $\mathbf{P}_{k, \text { SP }}$ must be estimated from $\hat{\mathbf{R}}_{k, p p}$. If the ISNR $\gg 1$, this can be done by partitioning its eigenvectors

$$
\begin{aligned}
\hat{\mathbf{R}}_{k, p p} \hat{\mathbf{U}} & =\hat{\mathbf{U}} \hat{\mathbf{\Lambda}} \\
\hat{\mathbf{U}}^{s} & =\left[\hat{\mathbf{u}}_{Q+1}, \cdots, \hat{\mathbf{u}}_{M_{p}}\right] \\
\mathbf{P}_{k, \mathrm{SP}} & =\left[\hat{\mathbf{U}}^{s}\left(\hat{\mathbf{U}}^{s}\right)^{H}, \mathbf{0}_{M_{a}}\right]
\end{aligned}
$$

where the eigenvalues in diagonal matrix $\hat{\boldsymbol{\Lambda}}$ are sorted in descending magnitude, $\hat{\mathbf{U}}$ is unitary, and $\mathbf{0}_{M_{a}}$ is an $M_{p} \times M_{a}$ matrix of zeros. The ${ }^{\wedge}$ designation is used on all eigen decomposition terms to distinguish these estimated values from the true interference subspace terms used in Section IV. It is assumed that some order estimation procedure such as the minimum description length (MDL) is used to identify Q [30]. It should be noted that this eigenvector partitioning approach relies on an assumption that noise is spatially white, i.e. that $\mathbf{R}^{\eta}=\sigma_{\eta}^{2} \mathbf{I}$. This ensures $\hat{\mathbf{U}}^{s}$ is not biased by noise, which is of particular concern in the RA environment where noise power typically exceeds signal power by orders of magnitude. The $k$ th short-term integration signal covariance estimate (i.e., $\hat{\mathbf{R}}_{k, p p}^{s}$ for the SP algorithm) is given by

$$
\hat{\mathbf{R}}_{k, \mathrm{SP}}^{s}=\mathbf{P}_{k, \mathrm{SP}} \hat{\mathbf{R}}_{k} \mathbf{P}_{k, \mathrm{SP}}
$$

Long-term integration and projection bias removal is accomplished using (9).

\section{Subspace Projection With Auxiliary Antennas (SPA)}

In this approach, the subspace projection method described above is applied with minor modification to the full augmented array, including auxiliaries. Special handling is required to spatially prewhiten the noise to avoid eigenvector bias by $\mathbf{R}^{\eta}$ because the auxiliaries will have higher noise level than the primaries. ${ }^{2}$ We must also ensure that the cross correlations between primary and auxiliary antennas do not appear in the final signal covariance estimate. These correlations have higher noise levels and contain no signal information; therefore, corresponding rows in the projection matrix are simply truncated. The eigenvectors of the full array prewhitened sample covariance are partitioned, and the filter matrix $\mathbf{P}_{k, \mathrm{SPA}}$ is formed as

$$
\begin{aligned}
\hat{\mathbf{R}}_{k}^{W} \hat{\mathbf{U}} & =\hat{\mathbf{U}} \hat{\mathbf{\Lambda}} \\
\mathbf{U}^{s} & =\left[\mathbf{u}_{Q+1}, \cdots, \mathbf{u}_{M}\right] \\
\mathbf{P}_{k, \mathrm{SPA}} & =\mathbf{I}_{M_{p}} \hat{\mathbf{U}}^{s}\left(\hat{\mathbf{U}}^{s}\right)^{H} \\
\mathbf{I}_{M_{p}} & =[\mathbf{I}, \mathbf{0}],: M_{p} \times M
\end{aligned}
$$

where $\hat{\mathbf{R}}_{k}^{W}=\left(\hat{\mathbf{R}}^{\eta}\right)^{-1 / 2} \hat{\mathbf{R}}_{k}\left(\hat{\mathbf{R}}^{\eta}\right)^{-1 / 2}$ is the noise-whitened full-augmented array sample covariance, $\hat{\mathbf{R}}^{\eta}$ is a sample estimate of noise alone (taken during a separate interference free time interval), and $\mathbf{I}_{M_{p}}$ is a truncated identity matrix used to

\footnotetext{
${ }^{2}$ The authors wish to acknowledge the contribution of manuscript reviewers who suggested this improvement.
} 
remove the last $M_{a}$ rows in computing $\mathbf{P}_{k, \mathrm{SPA}}$. The signal covariance estimate is given by

$$
\hat{\mathbf{R}}_{k, \mathrm{SPA}}^{s}=\mathbf{P}_{k, \mathrm{SPA}} \hat{\mathbf{R}}_{k}^{W} \mathbf{P}_{k, \mathrm{SPA}}^{H}
$$

Note that $\mathbf{P}_{k, \mathrm{SPA}}$ is not square and, therefore, is not strictly a projection matrix. This also requires a modification to the projection bias removal (9), so for SPA, we define

$$
\mathbf{C}=\sum_{k=0}^{K-1}\left[\mathbf{P}_{k, \mathrm{SPA}} \mathbf{I}_{M_{p}}^{T}\right]^{*} \otimes\left[\mathbf{P}_{k, \mathrm{SPA}} \mathbf{I}_{M_{p}}^{T}\right] .
$$

\section{Array Multiple Sidelobe Canceller (MSC)}

The classical multiple sidelobe canceller (MSC) [29] can be extended to form a projection-matrix-like operator that can be applied directly to $\hat{\mathbf{R}}$. This approach is related to the cross-spectral correlation post processing with a reference signal method of Briggs et al. [11]. Both use correlation estimates to compute the appropriate weight to scale subtraction of a high INR reference signal from the primary channel.

As originally conceived, a single MSC output is computed by subtracting the high ISNR auxiliary array signal from a single primary channel. The optimal MSC adaptive beamformer weight $\mathbf{w}_{m}^{*}$ for the $m$ th primary antenna is the minimum mean squared error solution given by

$$
\begin{aligned}
\mathbf{w}_{m}^{\star} & =\arg \min _{\mathbf{w}_{m}} E\left\{\left|y_{m}[n]-s_{m}[n]\right|^{2}\right\} \\
& =\mathbf{R}_{a a}^{-1}\left\{\mathbf{R}_{a p}\right\}\left(\operatorname{column}_{m}\right) \\
y_{m}[n] & =x_{p, m}[n]-\mathbf{w}_{m}^{H} \mathbf{x}_{a}[n], ; 1<m<M_{p} .
\end{aligned}
$$

Using the same auxiliary array in each case, a separate MSC beamformer can be computed for every primary element to produce an array MSC

$$
\mathbf{y}[n]=\left[\mathbf{I},-\mathbf{R}_{p a} \mathbf{R}_{a a}^{\dagger}\right] \mathbf{x}[n]
$$

where $\mathbf{y}[n]=\left[y_{1}[n], \cdots, y_{M_{p}}[n]\right]^{T}$. The pseudo inverse is used here to improve numerical stability when $Q<M_{a}$ and strong interferers produce high ISNR in the auxiliaries. Using (18)

$$
\begin{aligned}
\mathbf{R}_{y y} & =\mathbf{P R P}^{H}, \text { where } \\
\mathbf{P} & =\left[\mathbf{I},-\mathbf{R}_{p a} \mathbf{R}_{a a}^{\dagger}\right] .
\end{aligned}
$$

Replacing the true second-order moments with sample statistics and recomputing the projection for each short-term integration, we define the MSC algorithm as

$$
\begin{aligned}
\hat{\mathbf{R}}_{k, \mathrm{MSC}}^{s} & =\hat{\mathbf{R}}_{k, y y}=\mathbf{P}_{k, \mathrm{MSC}} \hat{\mathbf{R}}_{k} \mathbf{P}_{k, \mathrm{MSC}}^{H}, \text { where } \\
\mathbf{P}_{k, \mathrm{MSC}} & =\left[\mathbf{I},-\hat{\mathbf{R}}_{k, p a} \hat{\mathbf{R}}_{k, a a}^{\dagger}\right] .
\end{aligned}
$$

To justify this approach, it must be demonstrated that $\mathbf{R}_{y y}$ is in fact a useful estimate of $\mathbf{R}^{s}$. It is easily shown that when $\mathbf{R}_{a a}$ is full rank, e.g. with noise, (19) becomes $\mathbf{R}_{y y}=\mathbf{R}_{p p}-$ $\mathbf{R}_{p a} \mathbf{R}_{a a}^{\dagger} \mathbf{R}_{a p}$. Using (2), assuming noise at primary and auxiliary arrays are mutually independent and the space source signal level at the auxiliaries is negligible (reasonable for the weak RA sources with auxiliaries steered to interference), this becomes

$$
\mathbf{R}_{y y}=\left[\mathbf{R}_{p p}^{s}+\mathbf{R}_{p p}^{i}+\mathbf{R}_{p p}^{\eta}\right]-\left[\mathbf{R}_{p a}^{i}\left(\mathbf{R}_{a a}^{i}+\mathbf{R}_{p p}^{\eta}\right)^{\dagger} \mathbf{R}_{a p}^{i}\right] .
$$

Using (10) and properties of the pseudo inverse, it can be shown that when $Q \leq M_{a}$ and $\mathbf{R}_{a a}^{i}$ is rank $Q$, then $\mathbf{R}_{p a}^{i}\left(\mathbf{R}_{a a}^{i}\right)^{\dagger} \mathbf{R}_{a p}^{i}=$ $\mathbf{R}_{p p}^{i}$. Thus, for large INR levels at the auxiliaries, the second term in (21) becomes

$$
\begin{aligned}
{\left[\mathbf{R}_{p a}^{i}\left(\mathbf{R}_{a a}^{i}+\mathbf{R}_{p p}^{\eta}\right)^{\dagger} \mathbf{R}_{a p}^{i}\right] } & \approx \mathbf{R}_{p p}^{i}, \text { so } \\
\mathbf{R}_{y y} & \approx \mathbf{R}_{p p}^{s}+\mathbf{R}_{p p}^{\eta} .
\end{aligned}
$$

Thus, $\mathbf{R}_{y y}$ is precisely the desired interference cancelled result, and the appropriate sample-based signal covariance estimate is given by (20). It should be noted that the MSC algorithm requires that the number of auxiliary antennas $M_{a}$ be greater than or equal to the number of distinct interfering sources. The longterm integration result is simply the average over short-term intervals

$$
\hat{\mathbf{R}}_{\mathrm{MSC}}^{s}=\frac{1}{K} \sum_{k=0}^{K-1} \hat{\mathbf{R}}_{k, \mathrm{MSC}}^{s} .
$$

Bias removal as in (9) is not needed for the MSC algorithm.

\section{E. Cross Subspace Projection (CSP)}

It can be argued that the two previous algorithms suffer from structural problems that unnecessarily introduce error in $\hat{\mathbf{R}}^{s}$. Low ISNR correlations in $\mathbf{R}_{k, p p}$ were included in computing $\mathbf{P}_{k, \mathrm{SPA}}$ and $\mathbf{P}_{k, \mathrm{MSC}}$. This increases interference subspace estimation error. In addition, $\hat{\mathbf{R}}^{s}$ was computed as the product of a filter matrix ( $\mathbf{P}_{k, \mathrm{SPA}}$ or $\mathbf{P}_{k, \mathrm{MSC}}$ ) and the full $\hat{\mathbf{R}}_{k}$. This can unnecessarily reintroduce interference if the spatial filter is not perfect because $\hat{\mathbf{R}}_{k}$ includes high interference-level terms from the auxiliaries. The cross subspace projection algorithm presented here avoids both of these problems.

The projection matrix is constructed from a partitioned SVD of cross correlation $\hat{\mathbf{R}}_{k, p a}$

$$
\begin{aligned}
\hat{\mathbf{R}}_{k, p a} & =\hat{\mathbf{U}} \hat{\Gamma} \hat{\mathbf{V}}^{H} \\
\hat{\mathbf{U}}^{s} & =\left[\hat{\mathbf{u}}_{Q+1}, \cdots, \hat{\mathbf{u}}_{M_{p}}\right] \\
\mathbf{P}_{k, \mathrm{CSP}} & =\left[\hat{\mathbf{U}}^{s}\left(\hat{\mathbf{U}}^{s}\right)^{H}, \mathbf{0}_{M_{a}}\right]
\end{aligned}
$$

where $\hat{\Gamma}$ is diagonal with elements $\hat{\gamma}_{m}$, which are the singular values of $\hat{\mathbf{R}}_{k, p a}$, and $\hat{\mathbf{U}}$ and $\hat{\mathbf{V}}$ are the unitary singular vector matrices. The signal covariance estimate is computed as

$$
\hat{\mathbf{R}}_{k, \mathrm{CSP}}^{s}=\mathbf{P}_{k, \mathrm{CSP}} \hat{\mathbf{R}}_{k, p p} \mathbf{P}_{k, \mathrm{CSP}}^{H} .
$$

Note that since noise and signal are far below interference levels in the auxiliary channels, the interference subspace of $\hat{\mathbf{R}}_{k, p a}$ can be identified with less estimation error. Due to the $\mathbf{0}_{M_{a}}$ term in $\mathbf{P}_{k, \text { CSP }}$, only primary channel correlations $\hat{\mathbf{R}}_{k, p p}$, rather than the full $\hat{\mathbf{R}}_{k}$, are operated on by the projection matrix, thus eliminating leakage of interference from the auxiliaries into $\hat{\mathbf{R}}_{k, \mathrm{CSP}}^{s}$. $\mathbf{P}_{k, \mathrm{CSP}}$ is shown in Section IV to form a tight perpendicular 
subspace projection for interference and in Section $\mathrm{V}$ to have the best overall performance of the four algorithms. As with the MSC algorithm, it is required for CSP that $M_{a} \geq Q$. Long-term integration and projection bias removal are accomplished with (9).

\section{Subspace Estimation ACCURACy FOR SP AND CSP ALGORITHMS}

Mitigation effectiveness clearly depends on the accuracy of interference subspace estimates. A comparative analysis using perturbation theory and numerical simulation will be presented to assess subspace bias in the SP and CSP algorithms. The following results consider only sky signal and finite sample errorinduced bias terms in the short-term integration interference subspace estimates used to compute $\mathbf{P}_{k, \text { SP }}$ and $\mathbf{P}_{k, \text { CSP }}$. Projection bias in $\hat{\mathbf{R}}_{k}^{s}$ due to nonorthogonality of $\mathbf{R}^{s}$ and $\mathbf{R}^{i}$ is a separate matter that can arise even with perfect interference subspace estimation and is countered by the bias correction step of (9). On the other hand, compensating for subspace estimation bias is problematic because the filter matrices will not effectively block the interference, and this cannot be corrected by (9). Bias due to subspace smearing from interference motion during the short-term integration is also not considered here. Another approach to performance analysis of subspace projection interference suppression is found in [18], whereas [31] gives insight into limitations in cancellation for MSC-like algorithms.

\section{A. Arbitrary Sky Source With Multiple Interferers}

Let $\hat{\mathbf{U}}^{i}=\left[\hat{\mathbf{u}}_{1}, \cdots, \hat{\mathbf{u}}_{Q}\right]$ be the estimated interference subspace partition for either the SP or CSP algorithm using (13) or (25), respectively. Define $\mathbf{U}^{i}=\left[\mathbf{u}_{1}, \cdots, \mathbf{u}_{Q}\right]$ as the true interference subspace corresponding to the eigen decomposition of $\mathbf{R}_{p p}^{i}$ or left singular value decomposition of $\mathbf{R}_{p a}^{i}$. The subspace perturbation, or bias, in the $m^{t h}$ vector is

$$
\begin{aligned}
\mathbf{e}_{m} & \triangleq \hat{\mathbf{u}}_{m}-\mathbf{u}_{m}, \quad 1 \leq m \leq Q \\
& =\mathbf{e}_{m}^{s}+\mathbf{e}_{m}^{N} .
\end{aligned}
$$

where " $s$ " and " $N$ " denote biases due to signal $\mathbf{R}^{s}$ and finite sample length error $\mathbf{E}_{k}$ [see (7)], respectively. The second line represents a simplifying assumption that these two error terms are independent, and their effects are additive. If the noise is independent across array elements (a reasonable assumption for the widely spaced antennas in an imaging array), then $\mathbf{R}^{\eta}$ is diagonal and induces no bias terms directly but will affect $\mathbf{e}_{m}^{N}$. Simulation results are presented below that demonstrate these approximations do not significantly affect performance prediction accuracy.
Given knowledge of (or a model for) $\mathbf{R}^{s}, \mathbf{R}^{i}$, and $\mathbf{R}^{\eta}, \mathbf{e}_{m}^{s}$ can be computed deterministically, but $\mathbf{e}_{m}^{N}$ is random and requires a statistical analysis. Using eigenvector perturbation theory [32, pp. 1095-1104] and treating $\mathbf{R}^{s}$ as a relatively small perturbation on $\mathbf{R}^{i}$, it can be shown that for the SP algorithm, the $m$ th eigenvector signal perturbation is

$$
\mathbf{e}_{m, \mathrm{SP}}^{s} \approx \sum_{l \neq m}^{M_{p}} \frac{\mathbf{u}_{l}^{H} \mathbf{R}_{p p}^{s} \mathbf{u}_{m}}{\lambda_{l}-\lambda_{m}} \mathbf{u}_{l}
$$

where $\lambda_{l}$ is the $l^{t h}$ true eigenvalue of $\mathbf{R}_{p p}^{i}$.

For the CSP algorithm, a corresponding perturbation analysis of the singular value decomposition of $\mathbf{R}_{p a}^{i}$ perturbed by $\mathbf{R}_{p a}^{s}$ is developed along the lines of [33] to yield

$$
\mathbf{e}_{m, \mathrm{CSP}}^{s} \approx \sum_{l \neq m}^{M_{p}} \frac{\gamma_{m} \mathbf{u}_{l}^{H} \mathbf{R}_{p a}^{s} \mathbf{v}_{m}+\gamma_{l} \mathbf{v}_{l}^{H} \mathbf{R}_{a p}^{s} \mathbf{u}_{m}}{\gamma_{m}^{2}-\gamma_{l}^{2}} \mathbf{u}_{l}
$$

where $\gamma_{l}, \mathbf{u}_{l}$, and $\mathbf{v}_{l}$ are the true singular values and left and right singular vectors of $\mathbf{R}_{p a}^{i}$, respectively.

Kaveh and Barabell [34] expanded on results in [35] to develop an asymptotic statistical analysis of eigenvector perturbations due to sampling error for autocovariance matrices. This approach is applicable for evaluating the expected length of $\mathbf{e}_{m}^{N}$ in the SP algorithm. ${ }^{3}$ It was shown that for large $N$

$$
E\left\{\left\|\mathbf{e}_{m, \mathrm{SP}}^{N}\right\|_{2}^{2}\right\}=E\left\{\left(\mathbf{e}_{m}^{N}\right)^{H} \mathbf{e}_{m}^{N}\right\} \approx \frac{\lambda_{m}}{N} \sum_{l \neq m}^{M_{p}} \frac{\lambda_{l}}{\left(\lambda_{m}-\lambda_{l}\right)^{2}} .
$$

A new expression analogous to (29) is needed for the CSP algorithm. It is shown in Appendix A that for a singular value decomposition of a sample cross covariance matrix estimate, we have (30), shown at the bottom of the page, where $\operatorname{Re}\{\cdot\}$ indicates the real part, and it is stipulated that for $k>M_{a}$, $\mathbf{v}_{l}=\mathbf{0}$, and $\gamma_{l}=0$.

\section{B. Solution for a Single Interferer}

For the important case of a single interferer, $Q=1$, and only the first perturbation vector $\mathbf{e}_{1}=\mathbf{e}_{1}^{s}+\mathbf{e}_{1}^{N}$ is of interest. The true interference subspace is $\mathbf{u}_{1}=\mathbf{a}_{p} /\left\|\mathbf{a}_{p}\right\|_{2}$ [see (10)]. The $M_{p}$-dimensional subspace separation angle $\Theta$ may be used as a metric to quantify estimation error with a single parameter

$$
\Theta=\cos ^{-1}\left(\frac{\left|\mathbf{a}_{p}^{H}\left(\mathbf{a}_{p}+\mathbf{e}_{1}^{s}+\mathbf{e}_{1}^{N}\right)\right|}{\left\|\mathbf{a}_{p}\right\|_{2}\left\|\mathbf{a}_{p}++\mathbf{e}_{1}^{s}+\mathbf{e}_{1}^{N}\right\|_{2}}\right)
$$

${ }^{3}$ The contribution to sample error due to $\mathbf{R}^{s}$ is neglected here since signal power is assumed to be less than either noise or interference levels, and direct signal bias was handled separately in (27) and (28).

$$
\begin{aligned}
E\left\{\left\|\mathbf{e}_{m, \mathrm{CSP}}^{N}\right\|_{2}^{2}\right\} & \\
& \approx \frac{1}{N} \sum_{l \neq m}^{M_{p}} \frac{\left[\gamma_{m}^{2} \mathbf{u}_{l}^{H} \mathbf{R}_{p p} \mathbf{u}_{l} \mathbf{v}_{m}^{H} \mathbf{R}_{a a} \mathbf{v}_{m}+\gamma_{l}^{2} \mathbf{u}_{m}^{h} \mathbf{R}_{p p} \mathbf{u}_{m} \mathbf{v}_{l}^{H} \mathbf{R}_{a a} \mathbf{v}_{l}+2 \gamma_{m} \gamma_{l} \operatorname{Re}\left\{\mathbf{u}_{l}^{H} \mathbf{R}_{p p} \mathbf{u}_{m} \mathbf{v}_{l}^{H} \mathbf{R}_{a a} \mathbf{v}_{m}\right\}\right]}{\left(\gamma_{m}^{2}-\gamma_{l}^{2}\right)^{2}}
\end{aligned}
$$


Neglecting the low-level $\mathbf{R}^{s}$ terms and assuming noise is independent across the array yields the following expressions for the pertinent covariance matrices:

$$
\mathbf{R}^{\eta}=\left[\begin{array}{cc}
\sigma_{\eta, p}^{2} \mathbf{I}_{p} & \mathbf{0} \\
\mathbf{0} & \sigma_{\eta, a}^{2} \mathbf{I}_{a}
\end{array}\right]
$$

where $\mathbf{I}_{p}$ and $\mathbf{I}_{a}$ are $M_{p} \times M_{p}$ and $M_{a} \times M_{a}$ identity matrices, respectively, and $\sigma_{p, \eta}^{2}$ and $\sigma_{a, \eta}^{2}$ are noise power levels seen at primary and auxiliary antenna feeds, respectively. For a single interferer, $\mathbf{R}_{p p}=\sigma_{i, p}^{2} \mathbf{a}_{p} \mathbf{a}_{p}^{H}+\sigma_{\eta, p}^{2} \mathbf{I}_{p}$, which has eigenvalues $\lambda_{1}=\left\|\mathbf{a}_{p}\right\|_{2}^{2} \sigma_{i, p}^{2}+\sigma_{\eta, p}^{2}$ and $\lambda_{l \neq 1}=\sigma_{\eta, p}^{2}$. The auxiliary sub array auto and cross covariance are $\mathbf{R}_{a a} \stackrel{\eta, p}{=} \sigma_{i, a}^{2} \mathbf{a}_{a} \mathbf{a}_{a}^{H}+\sigma_{\eta, a}^{2} \mathbf{I}_{a}$, and $\mathbf{R}_{p a}=\sigma_{i, p} \sigma_{i, a} \mathbf{a}_{p} \mathbf{a}_{a}^{H}$. Singular value decomposition yields $\gamma_{1}=\sqrt{M_{p} M_{a}} \sigma_{i, p} \sigma_{i, a}, \gamma_{k \neq 1}=0$, and $\mathbf{v}_{1}=\mathbf{a}_{\mathbf{a}} /\left\|\mathbf{a}_{a}\right\|_{2}$. Assuming the INR at each primary element is $\sigma_{i, p}^{2} / \sigma_{\eta, p}^{2}$ implies that $\left\|\mathbf{a}_{p}\right\|_{2}^{2}=M_{p}$. Likewise, $\left\|\mathbf{a}_{a}\right\|_{2}^{2}=M_{a}$. Substitution into (29) leads to the simplified form

$$
E\left\{\left\|\mathbf{e}_{1, \mathrm{SP}}^{N}\right\|_{2}^{2}\right\} \approx \frac{\left(M_{p}-1\right)\left(\sigma_{\eta, p}^{4}+M_{p} \sigma_{i, p}^{2} \sigma_{\eta, p}^{2}\right)}{N M_{p}^{2} \sigma_{i, p}^{4}}
$$

and (30) produces

$$
E\left\{\left\|\mathbf{e}_{1, \mathrm{CSP}}^{N}\right\|_{2}^{2}\right\} \approx \frac{\left(M_{p}-1\right) \sigma_{\eta, p}^{2}\left(M_{a} \sigma_{i, a}^{2}+\sigma_{\eta, a}^{2}\right)}{N M_{p} M_{a} \sigma_{i, p}^{2} \sigma_{i, a}^{2}} .
$$

These equations provide the needed expected vector length, but the direction of $\mathbf{e}_{1}^{N}$ is unknown. To provide an upper bound on subspace separation $\Theta$, the worst case is assumed such that $\mathbf{e}_{1}^{N}$ is orthogonal to both $\mathbf{a}_{p}$ and the sky signal. Since a specific vector is needed to compute $\Theta$, the last eigen vector, or left singular vector $\mathbf{u}_{M_{p}}$, is chosen arbitrarily [see (12) and (24)]. This satisfies the orthogonality requirement. Thus, for purposes of evaluating (31)

$$
\mathbf{e}_{1}^{N}=\sqrt{E\left\{\left\|\mathbf{e}_{1}^{N}\right\|_{2}^{2}\right\}} \mathbf{u}_{M_{p}} .
$$

$\Theta$ can now be directly evaluated for an arbitrary $\mathbf{R}^{s}$ and a single interferer.

For a single point-source sky signal, it is possible to compute the eigen and singular value decompositions in closed form to give a simple expression for $\mathbf{e}_{1}^{s}$. It is shown in Appendix B that the exact point source perturbations are

$$
\mathbf{e}_{1, \mathrm{SP}}^{s}=\frac{1-\rho+\sqrt{1+\rho^{2}-2 \rho+4 \rho|w|^{2}}}{2 \rho w} \mathbf{d}_{p}
$$

for $w \neq 0$, and (37), shown at the bottom of the page, for $\varrho w+z \neq 0 . \mathbf{d}_{p}$ is the unit length primary array response in the source direction, $w=\mathbf{a}_{p}^{H} \mathbf{d}_{p}$, and $z=\mathbf{a}_{a}^{H} \mathbf{d}_{a} . \rho$ and $\varrho$ are ISRs at antenna feeds of the primary and auxiliary antennas, respectively (see Appendix B). When either denominator is zero, the signal and interference response vectors across the primary

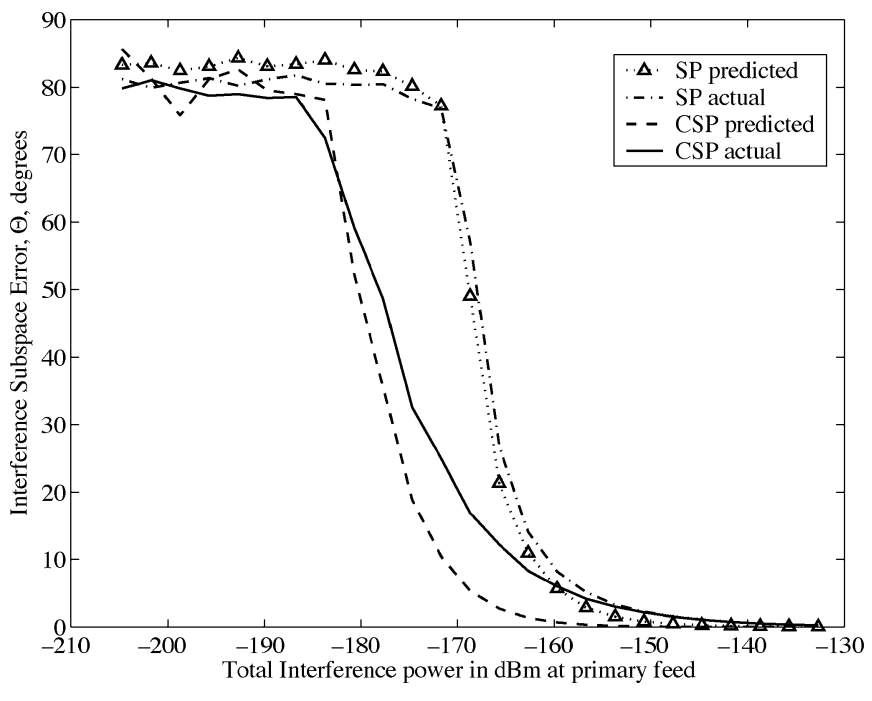

Fig. 3. Interference subspace estimation error angles for SP and CSP algorithms. Both Monte Carlo simulation and closed-form prediction equation results are presented for a single interferer case. SNR is $-22.5 \mathrm{~dB}$ at primary feed outputs for this $1 \mathrm{Jy}$ source.

or full arrays, respectively, are mutually orthogonal, and no bias occurs.

\section{Experimental Evaluation of Subspace Error}

The following experiment shows that for a practical signal scenario and reasonable auxiliary antenna designs $\Theta_{\mathrm{CSP}}<\Theta_{\mathrm{SP}}$. In other words, it helps in the mid-INR regime to use auxiliaries when computing the projection matrix $\mathbf{P}_{k}$. Fig. 3 illustrates this relationship by comparing $\Theta$ as a function of interferer power as predicted using (33) and (36) or (34) and (37) in (31). Predicted values are plotted with corresponding error found using a detailed Monte Carlo simulation of the sample-estimate-based SP and CSP algorithms. The simulation models a $1 \mathrm{Jy}\left(1 \mathrm{Jansky}=10^{-26} \mathrm{~W} / \mathrm{m}^{2} / \mathrm{Hz}\right)$ OH line source at $1.612 \mathrm{GHz}$, with system noise temperature of $35 \mathrm{~K}$ for the primaries and $90 \mathrm{~K}$ for auxiliary antennas. The VLA configuration of Fig. 1 was used, including two 3-m auxiliary antennas. The deep-space sky source was at $40^{\circ}$ azimuth (AZ) and $65^{\circ}$ elevation (EL), with the interferer at $25^{\circ} \mathrm{AZ}$ and $55^{\circ}$ EL. The modeled VLA primary element gains in the direction of interferer and space object source were, respectively, $g_{i}=0$ and $g_{s}=52 \mathrm{dBi}$ (dB relative to an isotropic antenna response). Corresponding gains for the auxiliary array antennas were $h_{i}=33.6$ and $h_{s}=-8.2 \mathrm{dBi}$. These physical parameters yield the following relative signal levels at the primary and auxiliary feeds: $\mathrm{SNR}_{p}=-22.5 \mathrm{~dB}, \mathrm{SNR}_{a}=-86.9 \mathrm{~dB}$, $\mathrm{INR}_{p}$, and $\mathrm{INR}_{a}$ are, respectively, 146 and $176 \mathrm{~dB}$ above the plotted interference $\mathrm{dBm}$ levels. We also define a normalized cross correlation-to-noise ratio XNR $=\left[\mathbf{R}_{p a}\right]_{(1,1)} /\left(\sigma_{\eta, p} \sigma_{\eta, a}\right)$. Due to low signal power and noise independence across the array, XNR is $191 \mathrm{~dB}$ above the plotted values of interference

$$
\mathbf{e}_{1, \mathrm{CSP}}^{s}=\frac{\left(1-\varrho^{2}+j 2 \varrho \operatorname{Im}\left\{z^{*} w\right\}+\sqrt{1+\varrho^{4}+2 \varrho^{2} \operatorname{Re}\left\{\left(z w^{*}\right)^{2}\right\}+4\left(\varrho+\varrho^{3}\right) \operatorname{Re}\left\{z w^{*}\right\}-2 \varrho^{2}\left(|z|^{2}|w|^{2}-2|w|^{2}-2|z|^{2}+1\right)}\right)}{2 \varrho^{2} w+2 \varrho z} \mathbf{d}_{p}
$$


$\mathrm{dBm}$ levels to within $0.3 \mathrm{~dB}$ across the range. Analysis is for a single short-term integration of $20 \mathrm{~ms}$, which corresponds to $N=2000$ at the $10^{5}$ sample/second baseband sample rate. Results are averaged over 20 trials.

It can be seen in Fig. 3 that the prediction equations reasonably match the Monte Carlo results for both SP and CSP. Due to the asymptotic nature of the analysis, much tighter fits occur for larger $N$, but this leads to unrealistic short-term integration times given interferer motion. Prediction accuracy also holds over variations in the number of auxiliary antennas and sky signal power levels.

\section{Simulation Results}

This section presents a series of simulations for different signal scenarios to compare interference removal performance for the four algorithms described above. In each case, the modeled telescope array is the full VLA in the configuration of Fig. 1 or subsets of these antennas. The desired source is a Hydroxyl ion $(\mathrm{OH})$ emission at $1612 \mathrm{MHz}$, and interference is modeled as the spectral sidelobes of a GLONASS satellite transmission. Processing bandwidth is $5.0 \mathrm{kHz}$ to satisfy the narrowband array assumption with respect to the interferer. The complex I-Q baseband data sample rate is $100 \mathrm{kHz}$, and the number of interferers $Q$ is assumed known.

Realistic models were used for antenna sidelobe response patterns in magnitude and phase, pattern variation among antennas, interfering satellite orbital motion and signal levels, correlation estimation smoothing caused by motion during short-term integration, system noise temperature, aperture efficiency, and other parameters required to produce simulation results representative of real data experiments. Particular attention was paid to accurately modeling antenna pattern sidelobe levels because, as seen in Appendix B, improved performance for the auxiliary assisted algorithms depends on the difference between ISR at feeds of the primary and auxiliary antennas. At a primary, interference is observed in the deep sidelobes. Simply using ideal circular aperture responses results in unrealistically low primary antenna sidelobes and, thus, would yield better interference rejection than can be achieved in practice.

Two performance measures are used to compare algorithm interference mitigation. First, we define a normalized mean square error in $\hat{\mathbf{R}}^{s}$ as

$$
\varepsilon_{s}^{2}=\frac{\frac{1}{L} \sum_{l=1}^{L}\left\|\mathbf{M} \odot\left(\hat{\mathbf{R}}_{l}^{s}-\mathbf{R}^{s}\right)\right\|_{F}^{2}}{\left\|\mathbf{M} \odot \mathbf{R}^{s}\right\|_{F}^{2}}
$$

where subscript $F$ indicates the Frobenius norm, $L$ is the number of Monte Carlo simulation trials, $\odot$ indicates element-wise Schur matrix product, and $\mathbf{M}$ is a masking matrix of all ones except zeros down the diagonal. Multiplying by $\mathbf{M}$ masks the diagonal elements that are dominated by $\mathbf{R}^{\eta}$ and are not used in radio imaging. Since an RA image is computed directly from $\mathbf{M} \odot \hat{\mathbf{R}}^{s}$, this metric is a measure of achievable image quality. $\varepsilon_{s}^{2}$ represents error power as a fraction of total power in $\mathbf{R}^{s}$.

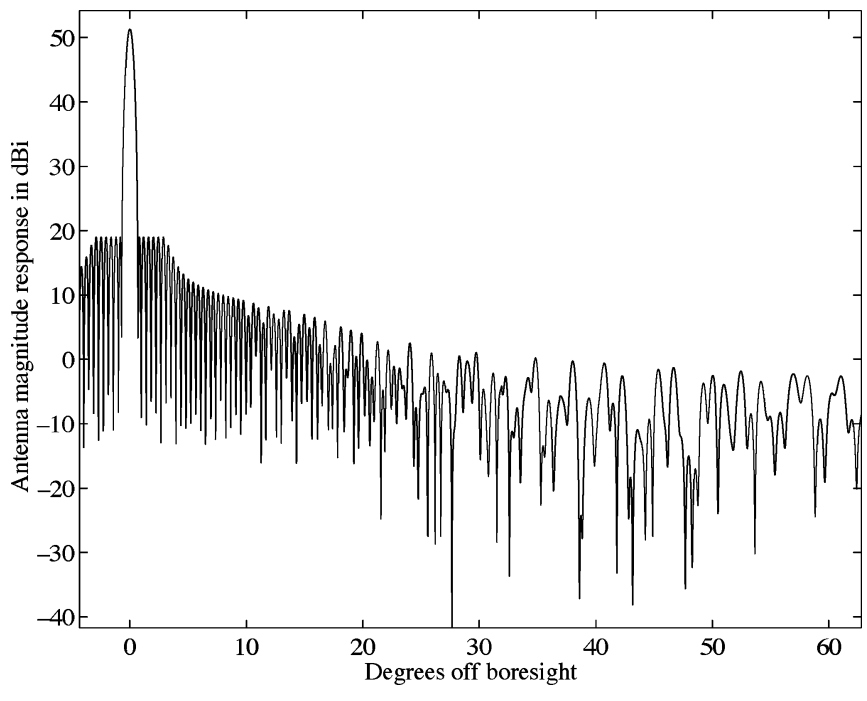

Fig. 4. Modeled antenna response for the VLA 25-m dishes. TICRA PTD results were manually scaled to match the measured sidelobe response envelope.

A second metric is the average (over short-term integration intervals) signal-to-interference power ratio at the filter output.

$$
\mathrm{SIR}=\frac{\operatorname{Tr}\left\{\sum_{k=0}^{K-1} \mathbf{P}_{k} \mathbf{R}^{s} \mathbf{P}_{k}^{H}\right\}}{\operatorname{Tr}\left\{\sum_{k=0}^{K-1} \mathbf{P}_{k} \mathbf{R}_{k}^{i} \mathbf{P}_{k}^{H}\right\}}
$$

where $\mathbf{R}_{k}^{i}$ is the true interference covariance taken at a time snapshot midway through short-term integration interval $k$. This metric yields basic insight into how the $\mathbf{P}_{k}$ are performing in attenuating interference relative to signal attenuation.

The gain response used for the 25-m dishes is shown in Fig. 4 and was obtained by modifying the results of a commercial numerical RF analysis package (TICRA), which is based on the physical theory of diffraction (PTD). On axis gain is $52 \mathrm{dBi}$. The response was computed using a Gaussian feed illumination pattern with $-11 \mathrm{~dB}$ taper at the reflector rim. A random reflector surface perturbation of $0.25 \mathrm{~mm}$ peak to peak was applied (consistent with VLA specifications), and edge currents were included in the model. Feed supports, the gross deviation of the actual VLA surface from parabolic, and the subreflector system were not modeled. These TICRA simulated sidelobe gain patterns were lower than measured values; therefore, the sidelobes were scaled larger by 0 to $20 \mathrm{~dB}$ as a function of off-axis angle to match typical sidelobe envelope shape as measured at the VLA. ${ }^{4}$ The 3-m auxiliary antenna response was also computed using TICRA. They have an on-axis gain of $30 \mathrm{dBi}$, which is $22 \mathrm{~dB}$ below the primaries. System noise temperature for primaries was $35 \mathrm{~K}$, which is the L-band specification for the VLA, whereas $90 \mathrm{~K}$ was assumed for the inexpensive auxiliary channels. With $\mathrm{BW}=5 \mathrm{kHz}$, this corresponds to noise power levels of -146 and $-142 \mathrm{dBm}$, respectively, when reflected to the antenna feeds.

Fig. 5 presents SIR results for scenario one, which involves two stationary interferers and two 3-m dish auxiliary antennas

\footnotetext{
${ }^{4}$ The authors acknowledge the contribution of D. Mertely of the National Radio Astronomy Observatory, VLA, for providing detailed antenna pattern information.
} 


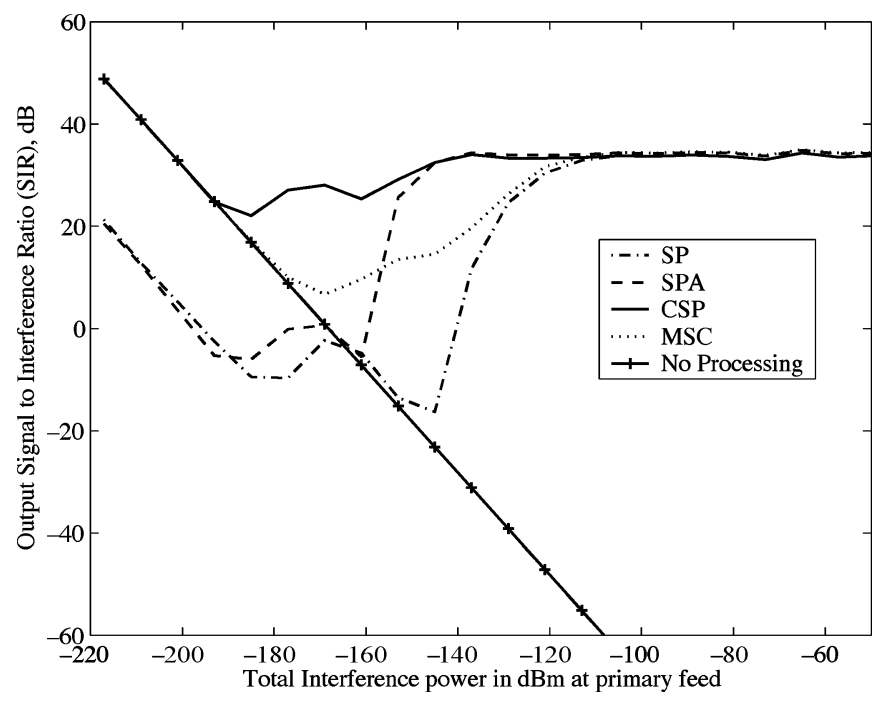

Fig. 5. SIR for two stationary interferers and two small auxiliary dish antennas aimed at the interferers. Source is $1 \mathrm{Jy} \mathrm{OH}$ emission. INR at primary feed outputs is $146 \mathrm{~dB}$ above the plotted $\mathrm{dBm}$ interferer power level. SNR is $-22.5 \mathrm{~dB}$ at primary feed outputs.

steered to them. Stationary interference is of course unrealistic except over the shortest of integration times but is used here to demonstrate the limiting best-case performance when subspace estimation error is not introduced by motion-induced temporal smearing of the interference array response. In this case, we cannot compute $\varepsilon_{s}^{2}$ since projection bias removal fails with no motion because $\mathbf{C}$ is singular. The full VLA array and auxiliaries (located at the two "x"s) of Fig. 1 were used. As shown in Fig. 6, the $\mathrm{OH}$ source is located at $20^{\circ} \mathrm{AZ}, 85^{\circ} \mathrm{EL}$ with flux density of $1 \mathrm{Jy}$, corresponding to $-169 \mathrm{dBm}$ at the primary feeds and below $-210 \mathrm{dBm}$ at the auxiliary feeds due to sidelobe attenuation. The interferers are at $30^{\circ} \mathrm{AZ}, 75^{\circ} \mathrm{EL}$, and $-15^{\circ} \mathrm{AZ}, 60^{\circ} \mathrm{EL}$, with the second interferer transmission level $20 \mathrm{~dB}$ below the first. These physical parameters correspond to signal levels of $\mathrm{SNR}_{p}=-22.5 \mathrm{~dB} ; \mathrm{SNR}_{a, 1}=-79.6$, and $\mathrm{SNR}_{a, 2}=-87.2 \mathrm{~dB}$, respectively, for the two auxiliaries, and $\mathrm{INR}_{p}, \mathrm{INR}_{a, 1}$, and $\mathrm{INR}_{a, 2}$ are, respectively, 146, 167, and 147 $\mathrm{dB}$ above the plotted interference $\mathrm{dBm}$ levels. Integration time is $10 \mathrm{~s}$ or $N=1.0 \times 10^{6}$ samples.

For a frame of reference, the interference power levels in $\mathrm{dBm}$ at the feeds can be compared to the detrimental levels specified by the International Telecommunication Union for RA in their recommendation ITU-R RA-769 [36]. For a single antenna telescope in the $1612-\mathrm{MHz}$ band, the ITU threshold power flux density level is $-238 \mathrm{dBW} / \mathrm{m}^{2} / \mathrm{Hz}$. Adjusting for $5-\mathrm{kHz}$ bandwidth, antenna surface area, feed efficiency, and assuming $0 \mathrm{dBi}$ sidelobes in the interfering direction, this is equivalent to $-193 \mathrm{dBm}$ at the primary feeds. The ITU detrimental level for the Very Long Baseline Array (VLBA) is $-208 \mathrm{dBW} / \mathrm{m}^{2} / \mathrm{Hz}$, which maps to $-163 \mathrm{dBm}$ at the feeds. Both ITU levels are in the low range of our performance analysis plots, which indicates that the studied algorithms will permit operation well beyond the ITU detrimental interference levels. Additionally, these levels assume 2000 seconds of integration, which increases interference attenuation by fringe rotation decorrelation as compared to our 10-s integration

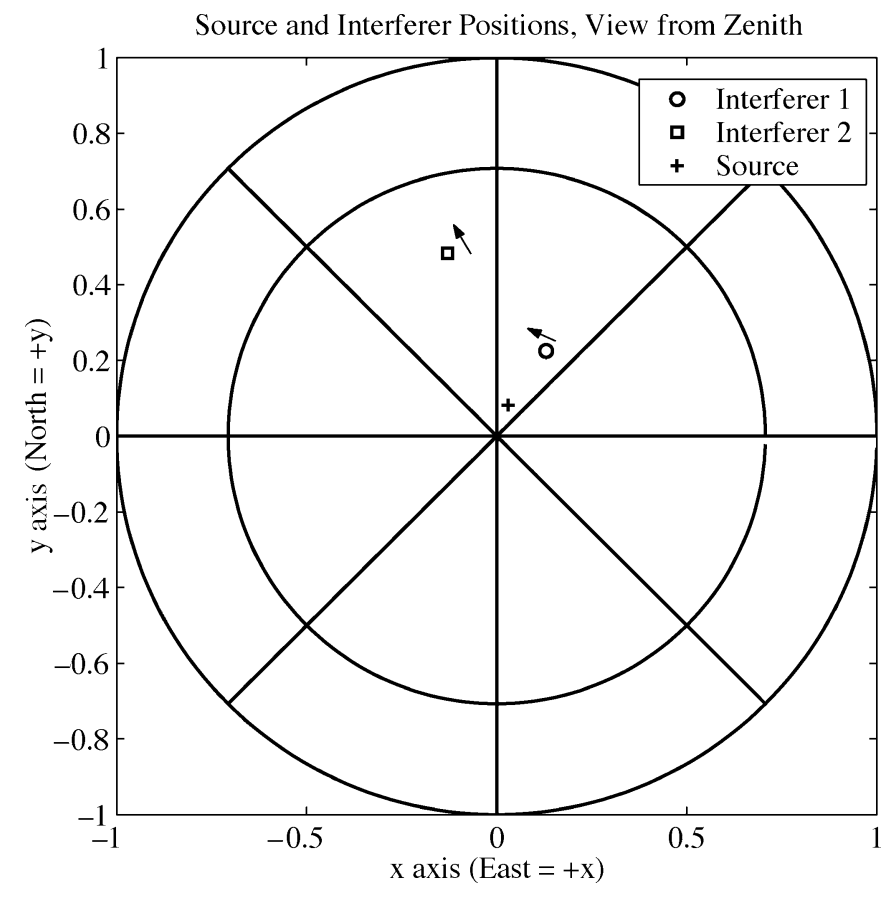

Fig. 6. Source geometry for a simulated $\mathrm{OH}$ radio source and two GLONASS interferers in scenarios one and two. Sky zenith is at the center of the graph, and the inner circle is $45^{\circ}$ elevation. Azimuth is relative to the $+y$ axis. Arrows indicate direction of orbital motion for scenario 2 .

time. Thus, for 10-s integration, interference power below the ITU recommendations would be detrimental, and use of the proposed algorithms is strongly indicated.

From Fig. 5, it can be seen that for mid-interference levels, all three auxiliary-assisted algorithms outperformed SP. At higher interference levels, all algorithms performed comparably. For interference power below $-160 \mathrm{dBm}, \mathrm{SP}$ and SPA appear to perform worse than no processing (however, in practice, these algorithms would be disabled in this range using the interference detector suggested in [13] and [14]). When SP or SPA is operated with very weak or no interference, the desired signal subspace is incorrectly detected as interference and excised. By using SP or SPA only when interference is detected, the problem is avoided. On the other hand, CSP and MSC need no detector and are seen here and in following figures to perform well with very low interference. All of these methods exhibit some subspace jitter due to covariance sample estimation error $\mathbf{E}_{k}$, as discussed in [28], which produces some variation in $\varepsilon_{s}^{2}$, as seen in Figs. 7 and 9.

Figs. 7 and 8 present results for a second scenario with two moving GLONASS satellites and two auxiliary antennas. Source and initial interferer locations are the same as scenario one, as shown in Fig. 6. Orbital angular velocity as seen at the array is $8 \times 10^{-4} \mathrm{o} / \mathrm{s}$ for each interferer, which is representative of a slower portion of the orbital arc for GLONASS. This experiment uses both auxiliaries and the center $M_{p}=12$ primary antennas of Fig. 1. Again, the $\mathrm{OH}$ source level is $1 \mathrm{Jy}$. Relative signal levels are $\mathrm{SNR}_{p}=-22.5 \mathrm{~dB}, \mathrm{SNR}_{a, 1}=-79.6$, and $\mathrm{SNR}_{a, 2}=$ $-87.2 \mathrm{~dB} . \mathrm{INR}_{p}, \mathrm{INR}_{a, 1}$, and $\mathrm{INR}_{a, 2}$ are respectively, 147, 171, and $149 \mathrm{~dB}$ above the plotted interference $\mathrm{dBm}$ levels. Longterm integration time is $10 \mathrm{~s}\left(N=1 \times 10^{6}\right)$, and short-term 


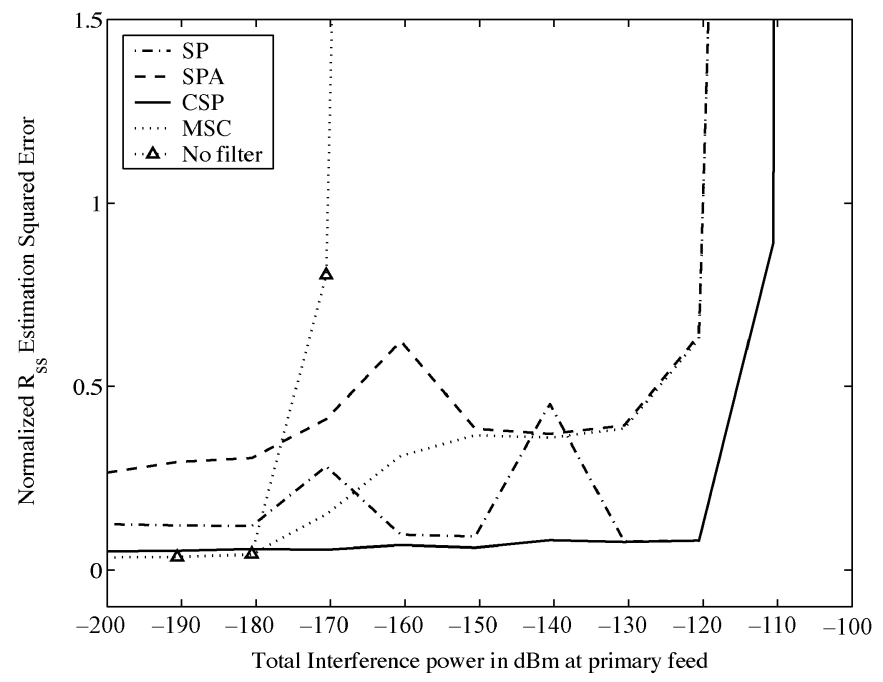

Fig. 7. Mean squared error in $\hat{\mathbf{R}}^{s}, \varepsilon_{s}^{2}$ for several algorithms in scenario 2, with two orbiting GLONASS interferers and two 3-m steered dish auxiliary antennas tracking the satellites. Source is $1 \mathrm{Jy} \mathrm{OH}$ emission. SNR is $-22.5 \mathrm{~dB}$ at primary feed outputs.

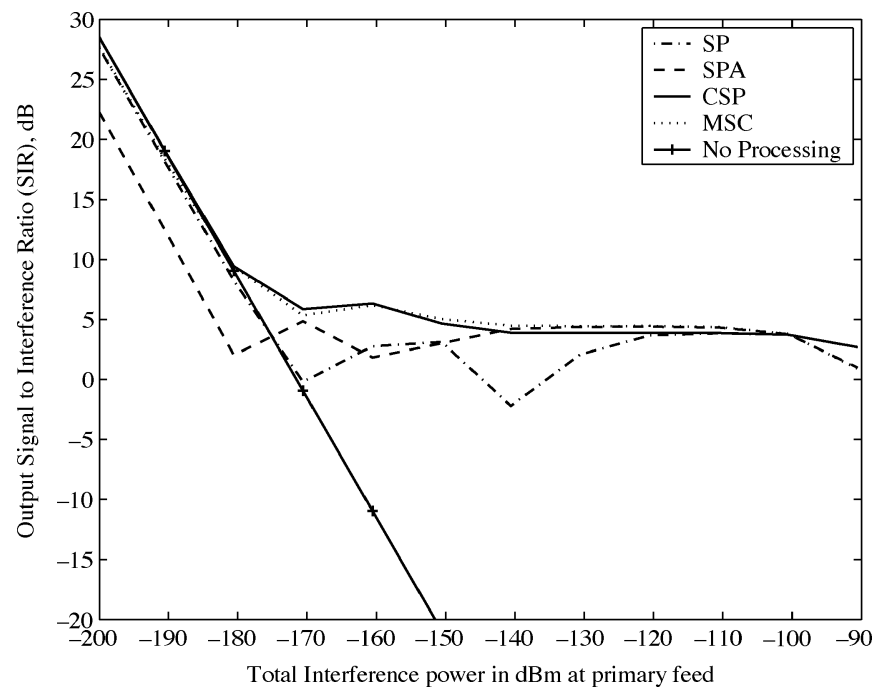

Fig. 8. SIR for scenario two. SNR is $-22.5 \mathrm{~dB}$ at primary feed outputs.

integration is $10 \mathrm{~ms}$ (1000 samples, $K=1000$.) This short interval was required to reduce subspace smearing and obtain acceptable interference rejection given satellite motion. During each short-term integration interval, tracking snapshots of the sample covariance matrices were recomputed three times and then averaged to simulate smearing.

It can be seen in Fig. 7 that CSP produced lower error estimates of $\hat{\mathbf{R}}^{s}$ for most interference power levels. The "no filtering" curve represents the achievable level of interference attenuation due to decorrelation of $\mathbf{R}^{i}$ during long-term integration over many motion induced interferometer "fringe rotations" in the array data, as described in [15]. All filtering methods outperformed "no filtering" at higher power levels. Although this example is typical, relative algorithm error ordering is scenario dependent. CSP is usually one of the lowest error algorithms, but SPA and MSC sometimes compete for that position. Fig. 8 shows that in terms of SIR, all methods using auxiliary antennas

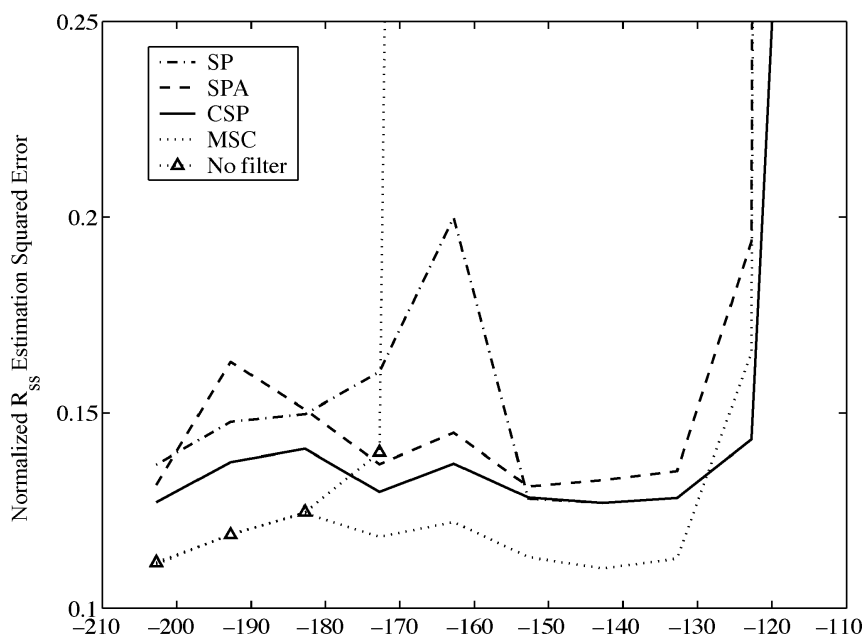

Fig. 9. Mean squared error in $\hat{\mathbf{R}}^{s}, \varepsilon_{s}^{2}$ for several algorithms in scenario two, with two orbiting GLONASS interferers and two 3-m steered dish auxiliary antennas tracking the satellites. Source is $1 \mathrm{Jy} \mathrm{OH}$ emission.

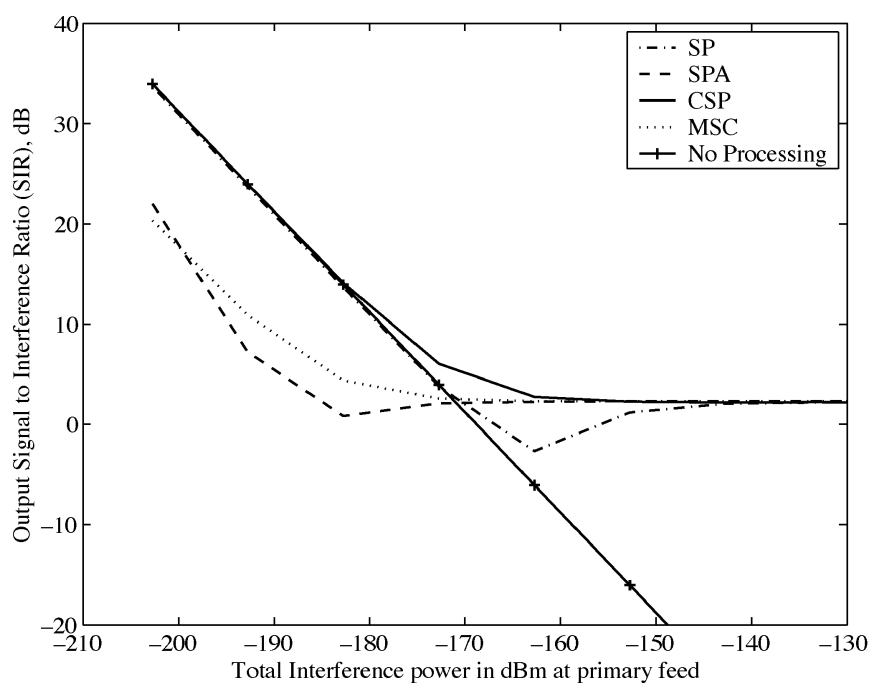

Fig. 10. SIR for scenario three. SNR is $-22.5 \mathrm{~dB}$ at primary feed outputs.

outperform SP. We have found that CSP is reliably the best performer in low to mid power regions and, thus, needs no interference detector to disable it.

Fig. 9 presents covariance error results for the third scenario, which includes a single moving GLONASS interferer and one 3-m dish auxiliary antenna steered to track. The array consists of the full nine element North-South branch and the four innermost antennas in each of the two lower branches of the VLA geometry of Fig. 1; therefore, $M_{p}=17$. Relative signal levels are $\mathrm{SNR}_{p}=-22.5 \mathrm{~dB}$ and $\mathrm{SNR}_{a, 1}=-84 \mathrm{~dB}$, whereas $\mathrm{INR}_{p}$ and $\mathrm{INR}_{a, 1}$ are, respectively, 146 and $185 \mathrm{~dB}$ above the plotted interference $\mathrm{dBm}$ levels. The desired source is at $-80^{\circ} \mathrm{AZ}, 65^{\circ}$ $\mathrm{EL}$ and initial interferer location is $85^{\circ} \mathrm{AZ}, 70^{\text {circ }} \mathrm{EL}$. Orbital speed is $0.008^{\circ} / \mathrm{s}$, which represents a faster transit than used in scenario two. Long-term integration time is $3 \mathrm{~s}\left(N=3 \times 10^{5}\right)$, and short-term integration is a very short $3 \mathrm{~ms}$ (300 samples, $K=1000$.) With the change in geometry and source motion, the relative order of lowest error algorithms is changed from scenario two. MSC has lowest error overall, with SPA and CSP doing better over some ranges of interference power. As before, 
"no filter" does provide some interference attenuation by decorrelation but is ineffective at higher INR levels. Fig. 10 shows that for this scenario, output SIR for CSP outperforms the other algorithms in the mid-range interference levels, whereas all algorithms converge to a constant SIR as INR gets very large.

\section{CONCLUSIONS}

The simulations presented above suggest that in the mid-interference power level regimes, use of high ISNR signals from auxiliary antennas will significantly improve interference removal. Subspace projection techniques perform much better with auxiliaries, and the MSC and CSP algorithms perform well-seamlessly from very low to very high interference levels.

We have already successfully tracked GLONASS and IRIDIUM satellite sources and computed real-time array correlations using three L-band 3-m az-el mount dish antennas, which are similar to the Small Radio Telescope (SRT) system introduced by MIT Haystack Observatory. Satellite orbital parameters and tracking control commands were generated by a low cost readily available software package "NOVA for Windows." This suggests that adding such an auxiliary antenna to an existing imaging array would not be prohibitively costly.

In order to effectively apply any of the methods described above, it will be necessary to add a short-term integrate-anddump capability to existing synthesis array correlator hardware. This is required to deal with satellite motion as in (9). To our knowledge, none of the major systems (e.g. VLA, Westerbork) currently have this ability, but new correlator development is in progress for the VLA. We recommend short-time integration dumps be incorporated in the design so that these powerful interference mitigation methods can be exploited.

\section{APPENDIX A}

An expression is developed for the mean squared length of the subspace perturbation vector due to finite sample estimation error in an SVD decomposition of a cross-covariance matrix. Let $\hat{\mathbf{R}}_{p a}=\mathbf{R}_{p a}+\mathbf{E}_{p a}$ be the sample cross covariance as defined in (5)-(7) (dropping the short-term integration index $k$ ). Let $\mathbf{u}_{m}$ and $\mathbf{v}_{m}$ be singular vectors of the true cross covariance such that $\mathbf{u}_{m}^{H} \mathbf{R}_{p a} \mathbf{v}_{m}=\gamma_{m}$. Let $\hat{\mathbf{u}}_{m}$ be the $m$ th left singular vector of $\hat{\mathbf{R}}_{p a}$, which is perturbed from the true $\mathbf{u}_{m}$ by $\mathbf{e}_{m}^{N}=\hat{\mathbf{u}}_{m}-\mathbf{u}_{m}$. It can be shown using matrix perturbation analysis (see [33] for a related development) that

$$
\mathbf{e}_{m}^{N} \approx \sum_{l \neq m}^{M_{p}} \frac{\gamma_{m} \mathbf{u}_{l}^{H} \mathbf{E}_{p a} \mathbf{v}_{m}+\gamma_{l} \mathbf{v}_{l}^{H} \mathbf{E}_{a p} \mathbf{u}_{m}}{\gamma_{m}^{2}-\gamma_{l}^{2}} \mathbf{u}_{l}
$$

Exploiting the fact that $\mathbf{u}_{l}$ are mutually orthogonal unit vectors, the expected perturbation vector norm squared is in (41), shown at the bottom of the page. It is assumed that $\mathbf{x}[n]$ is zero mean complex Gaussian, independent with respect to $n$. Thus, $N \hat{\mathbf{R}}$ has a Wishart distribution. Let $\mathbf{g}$ and $\mathbf{h}$ be arbitrary deterministic complex vectors. Noting that $E\{\hat{\mathbf{R}}\}=\mathbf{R}$ and $E\{\hat{\mathbf{E}}\}=\mathbf{0}$, it follows that

$$
\begin{aligned}
\operatorname{var}\left\{\mathbf{g}^{H} \hat{\mathbf{R}} \mathbf{h}\right\} & =E\left\{\left|\mathbf{g}^{H}(\mathbf{R}+\mathbf{E}) \mathbf{h}\right|^{2}\right\}-\left|\mathbf{g}^{H} \mathbf{R h}\right|^{2} \\
& =E\left\{\left|\mathbf{g}^{H} \mathbf{E h}\right|^{2}\right\} \\
& =\frac{1}{N} \mathbf{g}^{H} \mathbf{R} \mathbf{g h}{ }^{H} \mathbf{R h}
\end{aligned}
$$

where the final line follows due to the Wishart distribution as shown in [35, pp. 90 and 114]. Define $\mathbf{g}=\left[\mathbf{u}_{m}^{T}, 0, \cdots 0\right]^{T}$ and $\mathbf{h}=\left[0, \cdots 0, \mathbf{v}_{l}^{T},\right]^{T}$. Then, due to the block structure of $\mathbf{R}$ in (6)

$$
\begin{aligned}
E\left\{\left|\mathbf{g}^{H} \mathbf{E h}\right|^{2}\right\} & =E\left\{\left|\mathbf{u}_{m}^{H} \mathbf{E}_{p a} \mathbf{v}_{l}\right|^{2}\right\} \\
& =\frac{1}{N} \mathbf{u}_{m}^{H} \mathbf{R}_{p p} \mathbf{u}_{m} \mathbf{v}_{l}^{H} \mathbf{R}_{a a} \mathbf{v}_{l} .
\end{aligned}
$$

Substituting this and related terms (i.e., selecting different singular vectors as needed to define $\mathbf{g}$ and $\mathbf{h}$ ) into (42) yields (30).

\section{APPENDIX B}

In the single-interferer, noise-free case, the primary array autocovariance and the cross covariance with the auxiliary array can be modeled, respectively (neglecting a constant scale factor), as

$$
\begin{aligned}
& \mathbf{R}_{p p} \propto \mathbf{d}_{p} \mathbf{d}_{p}^{H}+\rho \mathbf{a}_{p} \mathbf{a}_{p}^{H} \\
& \mathbf{R}_{p a} \propto \mathbf{d}_{p} \mathbf{d}_{a}^{H}+\varrho \mathbf{a}_{p} \mathbf{a}_{a}^{H} .
\end{aligned}
$$

where $\mathbf{a}_{p}$ and $\mathbf{d}_{p}$ are the primary array responses (steering vectors) due to interference and deep space signal, respectively, and $\mathbf{a}_{a}$ and $\mathbf{d}_{a}$ are the corresponding response vectors at the auxiliary array. Primary and auxiliary feed ISRs are given by $\rho=\left(\sigma_{i}^{2} g_{i}^{2}\right) /\left(\sigma_{s}^{2} g_{s}^{2}\right)$ and $\varrho=\left(\sigma_{i}^{2} g_{i} h_{i}\right) /\left(\sigma_{s}^{2} g_{s} h_{s}\right)$, where $g_{i}$ and $g_{s}$ are the primary element gains in the direction of interferer and space object source, respectively, and $h_{i}$ and $h_{s}$ are the corresponding gains for the auxiliary array antennas. For a practical array configuration, $g_{i} \ll h_{i}$ and $g_{s} \gg h_{s}$; thus, $g_{i}^{2} / g_{s}^{2} \ll\left(g_{i} h_{i}\right) /\left(g_{s} h_{s}\right)$, and accordingly, $\rho \ll \varrho$.

Given the structure of $\mathbf{R}_{p p}$ from (44), when $Q=1$, the SP algorithm yields

$$
\hat{\mathbf{U}}^{i}=\hat{\mathbf{u}}_{1}=\mathbf{a}_{p}+\beta_{\mathrm{SP}} \mathbf{d}_{p} .
$$

The desired true subspace is $\mathbf{u}_{1}=\mathbf{a}_{p}$, and any nonzero $\beta_{\mathrm{SP}}$ represents an estimate perturbation in the direction of $\mathbf{d}_{p}$. The eigen equation is solved in closed form by substituting (44) and (46) into

$$
\begin{aligned}
\lambda \mathbf{u}_{\mathrm{SP}}= & \mathbf{R}_{p p} \mathbf{u}_{\mathrm{SP}}, \text { so } \\
\lambda\left(\mathbf{a}_{p}+\beta_{\mathrm{SP}} \mathbf{d}_{p}\right)= & \left(\mathbf{d}_{p} \mathbf{d}_{p}^{H}+\rho \mathbf{a}_{p} \mathbf{a}_{p}^{H}\right)\left(\mathbf{a}_{p}+\beta_{\mathrm{SP}} \mathbf{d}_{p}\right) \\
\lambda \mathbf{a}_{p}+\lambda \beta_{\mathrm{SP}} \mathbf{d}_{p}= & \left(\mathbf{a}_{p}\left(\rho\left\|\mathbf{a}_{p}\right\|^{2}+\rho \beta_{\mathrm{SP}} w\right)\right. \\
& \left.\quad+\mathbf{d}_{p}\left(w^{*}+\beta_{\mathrm{SP}}\left\|\mathbf{d}_{p}\right\|^{2}\right)\right)
\end{aligned}
$$

where $w=\mathbf{a}_{p}^{H} \mathbf{d}_{p}$. Matching terms yields $\lambda=\rho\left\|\mathbf{a}_{p}\right\|^{2}+$ $\rho \beta_{\mathrm{SP}} w$, and $\lambda \beta_{\mathrm{SP}}=w^{*}+\beta_{\mathrm{SP}}\left\|\mathbf{d}_{p}\right\|^{2}$. Assuming (without loss

$$
E\left\{\left\|\mathbf{e}_{m}^{N}\right\|_{2}^{2}\right\} \approx \sum_{l \neq m}^{M_{p}} \frac{\left[\gamma_{m}^{2} E\left\{\left|\mathbf{u}_{l}^{H} \mathbf{E}_{p a} \mathbf{v}_{m}\right|^{2}\right\}+\gamma_{l}^{2} E\left\{\left|\mathbf{u}_{m}^{H} \mathbf{E}_{p a} \mathbf{v}_{l}\right|^{2}\right\}+2 \gamma_{m} \gamma_{l} \operatorname{Re}\left\{E\left\{\mathbf{u}_{l}^{H} \mathbf{E}_{p a} \mathbf{v}_{m} \mathbf{u}_{m}^{H} \mathbf{E}_{p a} \mathbf{v}_{l}\right\}\right\}\right]}{\left(\gamma_{m}^{2}-\gamma_{l}^{2}\right)^{2}} .
$$


of generality) that array response vectors are all unit norm, and taking the largest magnitude root with respect to $\beta_{\mathrm{SP}}$ of these simultaneous equations, yields

$$
\beta_{\mathrm{SP}}=\frac{1-\rho+\sqrt{1+\rho^{2}-2 \rho+4 \rho|w|^{2}}}{2 \rho w}, \quad \text { for } w \neq 0 .
$$

When $w=0, \mathbf{a}_{p}$ and $\mathbf{d}_{p}$ are orthogonal so the eigen decomposition separates the two vectors, and no bias occurs. Substituting (48) into (46) leads to (36).

CSP subspace estimation error is evaluated similarly with a closed-form eigen analysis on $\mathbf{R}_{p a} \mathbf{R}_{p a}^{H}$ (i.e., the dominant left singular vector of $\mathbf{R}_{p a}$ is computed). Substituting (45) into $\gamma \mathbf{u}_{\mathrm{CSP}}=\mathbf{R}_{p a} \mathbf{R}_{p a}^{H} \mathbf{u}_{\mathrm{CSP}}$, where $\mathbf{u}_{\mathrm{CSP}}$, has the form $\mathbf{u}_{\mathrm{CSP}}=$ $\mathbf{a}_{p}+\beta_{\mathrm{CSP}} \mathbf{d}_{p}$, and matching terms as above leads to (37).

\section{REFERENCES}

[1] C. Barnbaum and R. Bradley, "A new approach to interference excision in radio astronomy: real-time adaptive cancellation," Astronom. J., vol. 116, pp. 2598-2614, Nov. 1998.

[2] J. B. S. W. Ellingson and J. Bunton, "Removal of the GLONASS C/A signal from $\mathrm{OH}$ spectral line observations using a parametric modeling technique," Astrophys. J. Suppl., vol. 135, pp. 87-93, July 2001.

[3] S. Ellingson, J. Bunton, and J. Bell, "Cancellation of glonass signals from radio astronomy data," in Proc. SPIE Conf. 4015, Munich, Germany, Mar. 2000, pp. 400-407.

[4] W. Combrinck, M. West, and M. Gaylard, "Coexisting with glonass: observing the $1612 \mathrm{mhz}$ hydroxyl line," Astron. Soc. Pacific, vol. 106, pp. 807-812, July 1994 .

[5] A. Leshem, A.-J. van der Veen, and E. Deprettere, "Detection and blanking of GSM interference in radio-astronomical observations," in Proc. Second IEEE Workshop Signal Process. Adv. Wireless Commun., 1999, pp. 374-377.

[6] A. Leshem and A.-J. van der Veen, "The effect of blanking of TDMA interference on radio-astronomical correlation measurements," in Proc. IEEE Signal Process. Workshop Higher Order Statist., 1999, pp. 25-29.

[7] P. Fridman and W. Baan, "RFI mitigation methods in radio astronomy," Astron. Astrophys., vol. 378, pp. 327-344, Oct. 2001.

[8] B. Smolders and G. Hampson, "Deterministic RF nulling in phased arrays for the next generation of radio telescopes," IEEE Antennas Propagat. Mag., vol. 44, no. 4, pp. 13-22, Aug. 2002.

[9] S. Ellingson, "Beamforming and interference canceling with very large wideband arrays," IEEE Trans. Antennas Propagat., vol. 51, no. 6, pp. 1338-1346, June 2003.

[10] A. Poulsen, "Real-Time Adaptive Cancellation of Satellite Interference in Radio Astronomy," Master's thesis, Brigham Young Univ., Provo, UT, Aug. 2003.

[11] F. Briggs, J. Bell, and J. Kesteven, "Removing radio interference from contaminated astronomical spectra using an independent reference signal and closure relations," Astronom. J., vol. 120, pp. 3351-3361, Aug. 2000.

[12] A. Leshem, A.-J. van der Veen, and A. Boonstra, "Multichannel interference mitigation techniques in radio astronomy," Astrophys. J. Suppl., vol. 131, no. 1, pp. 355-374, 2000.

[13] J. Raza, A.-J. Boonstra, and A.-J. van der Veen, "Spatial filtering of rf interference in radio astronomy," IEEE Signal Processing Lett., vol. 9, no. 2, pp. 64-67, Feb. 2002.

[14] A. Leshem and A.-J. van der Veen, "Radio-astronomical imaging in the presence of strong radio interference," IEEE Trans. Inf. Theory, vol. 46, no. 5, pp. 1730-1747, Aug. 2000.

[15] A. Thompson, "The response of a radio-astronomy synthesis array to interfering signals," IEEE Trans. Antennas Propag., vol. 30, no. 3, pp. 450-456, May 1982.

[16] B. Jeffs, K. Warnick, and L. Li, "Improved interference cancellation in synthesis array radio imaging using auxiliary antennas," in Proc. IEEE Int. Conf. Acoust, Speech, Signal Process., Hong Kong, Apr. 2003.

[17] R. Hung and R. Turner, "A fast beamforming algorithm for large arrays," IEEE Trans. Aerosp. Electron. Syst., vol. AES-19, no. 4, pp. 598-607, 1983.

[18] H. Subbaram and K. Abend, "Interference suppression via orthongonal projections: a performance analysis," IEEE Trans. Antennas Propagat., vol. 41, no. 9, pp. 1187-1194, Sep. 1993.
[19] L. Scharf and B. Friedlander, "Matched subspace detectors," IEEE Trans. Signal Processing, vol. 42, no. 8, pp. 2146-2157, Aug. 1994.

[20] A. Haimovich, "The eigencanceler: adaptive radar by eigenanalysis methods," IEEE Trans. Aerosp. Electron. Syst., vol. 32, no. 2, pp. 532-542, Apr. 1996.

[21] T. Palka and D. Tufts, "Reverberation characterization and suppressions by means of principal components," in Proc. IEEE Conf. Oceans, vol. 3, Sep. 1998, pp. 1501-1506.

[22] S. Won and K. Chong, "Robust adaptive beamforming based on the eigenstructure method," IEEE Trans. Signal Process., vol. 42, no. 6, pp. 1543-1547, Jun. 1994.

[23] B. Friedlander, "A signal subspace method for adaptive interference cancellation," IEEE Trans. Acoust., Speech, Signal Process., vol. 36, no. 12, pp. 1835-1845, Dec. 1988.

[24] R. Ekers and J. Bell, "Radio frequency interference," in Proc. IAU Symp. Universe Low Radio Frequencies, San Francisco, CA, Dec. 1999.

[25] J. Bell et al., "Software radio telescope: interference mitigation atlas and mitigation strategies," in Perspectives in Radio Astronomy: Scientific Imperatives at $\mathrm{cm}$ and $m$ Wavelengths, P. P. van Haarlem and J. M. van der Hulst, Eds. Wingeloo, The Netherlands: NFRA, 1999.

[26] A. Thompson, J. Moran, and G. Swenson Jr., Interferometry and Synthesis in Radio Astronomy, Second ed. New York: Wiley-Interscience, 2001.

[27] P. Napier, A. Thompson, and R. Ekers, "The very large array: design and performance of a modern synthesis radio telescope," Proc. IEEE, vol. 71, pp. 1295-1320, 1983.

[28] S. Ellingson and G. Hampson, "A subspace-tracking approach to interference nulling for phased array-based radio telescopes," IEEE Trans. Antennas Propag., vol. 50, no. 1, pp. 25-30, Jan. 2002.

[29] S. Applebaum and D. Chapman, "Adaptive arrays with main beam constraints," IEEE Trans. Antennas Propag., vol. AP-24, pp. 650-662, Sep. 1976.

[30] G. Schwartz, "Estimating the dimension of a model," Ann. Statist., vol. 6, pp. 461-464, 1978.

[31] S. Ellingson, "Capabilities and limitations of adaptive canceling for microwave radiometry," in Proc. IEEE Int. Geosci. Remote Sensing Symp., vol. 3, June 2002, pp. 1685-1687.

[32] C. Cohen-Tannoudji, B. Diu, and F. Laloë, Quantum Mechanics. New York: Wiley-Interscience, 1977

[33] A. Kot, Y. Lee, and H. Babri, "Bias and variance calculation of a principal component based frequency estimator," Proc. Inst. Elect. Eng. Vision Image Signal Process., vol. 142, no. 4, pp. 247-251, Aug. 1995.

[34] M. Kaveh and A. Barabell, "The statistical performance of MUSIC and the minimum-norm algorithms in resolving plane waves in noise," IEEE Trans. Acoust., Speech, Signal Process., vol. ASSP-34, no. 2, pp. 331-341, Apr. 1986.

[35] D. Brillinger, Time Series: Data Analysis and Theory, First ed. New York: Holt, Rinehart and Winston, 1975.

[36] Recommendation ITU-R RA.769-1, Protection Criteria Used for Radioastronomical Measurements, Int. Telecommun. Union, Geneva, Switzerland, 1995.

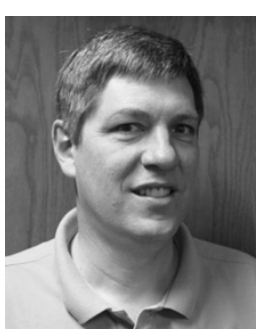

Brian D. Jeffs (M'90-SM'03) received B.S. and M.S. degrees in electrical engineering from Brigham Young University, Provo, UT, in 1978 and 1982, respectively, and the Ph.D. degree from the University of Southern California, Los Angeles, in 1989, also in electrical engineering.

Since 1990, he has been with the Department of Electrical and Computer Engineering, Brigham Young University, where he is currently an associate professor. He lectures in the areas of digital signal processing, digital image processing, and circuits. From 1982 to 1989, he was with Hughes Aircraft Company, Fullerton, CA, where he served as a sonar signal processing systems engineer in the anti-submarine warfare group. Current research interests include high data rate digital multiple antenna wireless communications systems, adaptive interference mitigation algorithms for radio astronomy, and blind image restoration.

Dr. Jeffs was a Vice General Chair for IEEE ICASSP-2001, Salt Lake City, UT. He was a member of the executive organizing committee for the 1998 IEEE DSP Workshop and served several years as chair of the Utah Chapter of the IEEE Communications and Signal Processing Societies. 


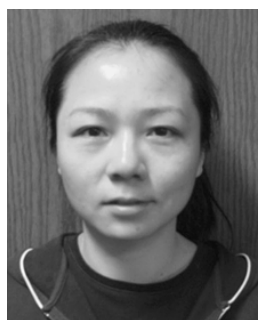

Lisha Li graduated from Chongqing University of Posts and Telecommunications, Chongqing, China, and received the M.S. degree in electrical engineering from Beijing University of Posts and Telecommunications, Bejing, China, in 1999. She is currently pursuing the Ph.D. degree in electrical engineering at Brigham Young University, Provo, UT.

Her research interests include array signal processing, image processing, and telecommunications.

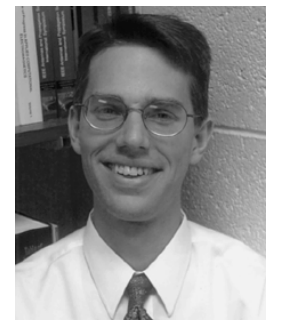

Karl F. Warnick (M'97) received the B.S. degree with University Honors in 1994 and the Ph.D. degree in 1997, both from Brigham Young University (BYU), Provo, UT.

From 1998 to 2000, he was a postdoctoral research associate and Visiting Assistant Professor with the Center for Computational Electromagnetics, University of Illinois at Urbana-Champaign. Since 2000, he has been an Assistant Professor with the Department of Electrical and Computer Engineering, BYU. His research interests include computational electromagnetics, rough surface scattering, remote sensing, inverse scattering, and numerical analysis. He has coauthored a book chapter and over 50 scientific journal papers and conference presentations.

Dr. Warnick was a recipient of the National Science Foundation Graduate Research Fellowship. 